\title{
Ideal simplices and double-simplices, their non-orientable hyperbolic manifolds, cone manifolds and orbifolds with Dehn type surgeries and graphic analysis
}

\author{
E. Molnár, I. Prok, and J. Szirmai®
}

To Memory of Professor Gyula (Julius) Strommer on the 100th Anniversary of His Birth

\begin{abstract}
In connection with our works in Molnár (On isometries of space forms. Colloquia Math Soc János Bolyai 56 (1989). Differential geometry and its applications, Eger (Hungary), North-Holland Co., Amsterdam, 1992), Molnár (Acta Math Hung 59(1-2):175-216, 1992), Molnár (Beiträge zur Algebra und Geometrie 38/2:261-288, 1997) and Molnár et al. (in: Prékopa, Molnár (eds) Non-Euclidean geometries, János Bolyai memorial volume mathematics and its applications, Springer, Berlin, 2006), Molnár et al. (Symmetry Cult Sci 22(3-4):435-459, 2011) our computer program (Prok in Period Polytech Ser Mech Eng 36(3-4):299$316,1992)$ found 5079 equivariance classes for combinatorial face pairings of the double-simplex. From this list we have chosen those 7 classes which can form charts for hyperbolic manifolds by double-simplices with ideal vertices. In such a way we have obtained the orientable manifold of Thurston (The geometry and topology of 3-manifolds (Lecture notes), Princeton University, Princeton, 1978), that of Fomenko-Matveev-Weeks (Fomenko and Matveev in Uspehi Mat Nauk 43:5-22, 1988; Weeks in Hyperbolic structures on three-manifolds. Ph.D. dissertation, Princeton, 1985) and a nonorientable manifold $M_{c^{2}}$ with double simplex $\widetilde{\mathcal{D}}_{1}$, seemingly known by Adams (J Lond Math Soc (2) 38:555-565, 1988), Adams and Sherman (Discret Comput Geom 6:135-153, 1991), Francis (Threemanifolds obtainable from two and three tetrahedra. Master Thesis, William College, 1987) as a 2-cusped one. This last one is represented for us in 5 non-equivariant double-simplex pairings. In this paper we are
\end{abstract}

E. Molnár's lecture "Algorithmic and Computational Aspects of Polyhedral Manifolds and Orbifolds" was held at "Internationale Tagung über Geometrie, Algebra und Analysis" 0105. Mai 1999, Balatonfüred (Ungarn). Gyula Strommer Memorial Conference, Eds. Karáné G, Sachs H, Schipp F, pp. 293-315. 
going to determine the possible Dehn type surgeries of $M_{c^{2}}=\widetilde{\mathcal{D}}_{1}$, leading to compact hyperbolic cone manifolds and multiple tilings, especially orbifolds (simple tilings) with new fundamental domain to $\widetilde{\mathcal{D}}_{1}$. Except the starting regular ideal double simplex, we do not get further surgery manifold. We compute volumes for starting examples and limit cases by Lobachevsky method. Our procedure will be illustrated by surgeries of the simpler analogue, the Gieseking manifold (1912) on the base of our previous work (Molnár et al. in Publ Math Debr, 2020), leading to new compact cone manifolds and orbifolds as well. Our new graphic analysis and tables inform you about more details. This paper is partly a survey discussing as new results on Gieseking manifold and on $M_{c^{2}}$ as well, their cone manifolds and orbifolds which were partly published in Molnár et al. (Novi Sad J Math 29(3):187-197, 1999) and Molnár et al. (in: Karáné, Sachs, Schipp (eds) Proceedings of "Internationale Tagung über geometrie, algebra und analysis", Strommer Gyula Nemzeti Emlékkonferencia, Balatonfüred-Budapest, Hungary, 1999), updated now to Memory of Professor Gyula Strommer. Our intention is to illustrate interactions of Algebra, Analysis and Geometry via algorithmic and computational methods in a classical field of Geometry and of Mathematics, in general.

Mathematics Subject Classification. Primary 57M50; Secondary 57N10.

Keywords. Hyperbolic manifold and cone manifold by fundamental polyhedron, Gieseking manifold and its analogue, Dehn type surgery, volume by Lobachevsky function.

\section{Introduction}

By pairing and logical gluing the side $(d-1)$-faces (i.e. facets) of an affine $d$-polytope $\mathcal{P}$, we can obtain a piecewise linear $d$-manifolds $\mathcal{M}$, where the fundamental group $\mathcal{G}$ of $\mathcal{M}$ will be finitely generated by the affine mappings $\mathcal{I}\left(\mathbf{g}_{1}, \mathbf{g}_{1}^{-1}, \mathbf{g}_{2}, \mathbf{g}_{2}^{-1}, \ldots\right)$ of paired facets. Thus, the $\mathcal{G}(\mathcal{I})$-equivalence classes of $(d-2)$-faces, called edges, will be induced, and for each edge class $\mathfrak{e}$ a (combinatorial) rotational order $\nu(\mathfrak{e})$ can be prescribed by so-called Poincaré algorithm [12]. Roughly speaking, we go round any edge $e$ from the edge class $\mathfrak{e}$ in a transversal 2-plane of the combinatorial tiling $\mathcal{T}:=(\mathcal{P}, \mathcal{G}(\mathcal{I}))$, where we meet all image edge-domains of the edges in class $\mathfrak{e}$, glued together. The repetition order $\nu(\mathfrak{e})$ will sign, when we turn back into the original identity domain of $\mathcal{P}$.

The collection $(\mathcal{P}, \mathcal{G}(\mathcal{I}, \nu(\mathfrak{e})))$ will define the universal covering space tiling $\mathcal{T}$ and the fundamental group $\mathcal{G}$ up to a presentation. In [12] we characterized in more details whether the tiling $\mathcal{T}$ is realizable in a simply connected homogeneous Riemann space. There occur some difficulties already in dimensions $d=3$, see also $[13,14]$.

These will be just illustrated by the Gieseking manifold and its related tiling in $\mathbb{H}^{3}$, furthermore by the 3 possible double-simplex manifolds and their exactly 7 nonequivariant tilings in $\mathbb{H}^{3}$. If the building simplex is regular with ideal 


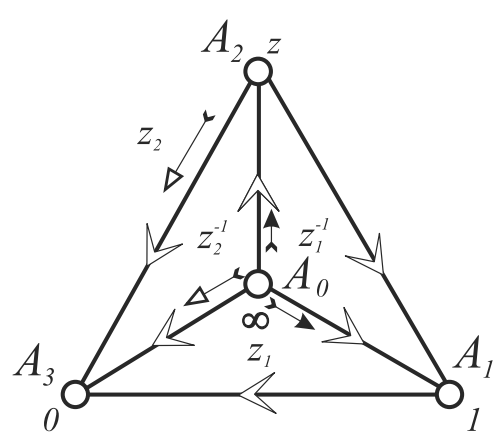

a

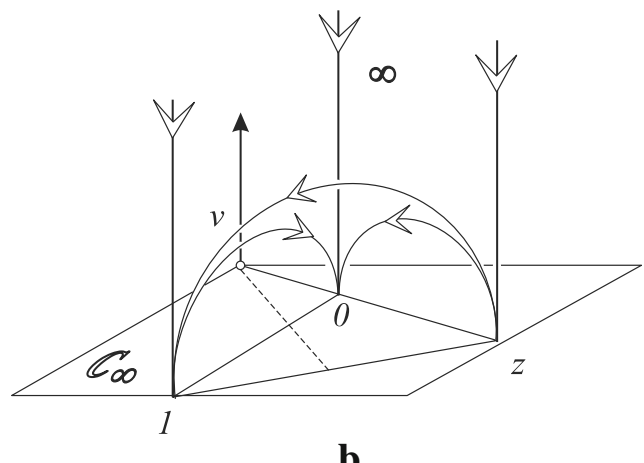

b

Figure 1 The Gieseking ideal simplex manifold: a with its symbolic Schlegel diagram; b the Poincaré half space model of hyperbolic space $\mathbb{H}^{3}$

vertices, then we obtain noncompact Riemann manifold $\mathcal{M}=\tilde{\mathcal{P}}=\mathbb{H}^{3} / \mathcal{G}$ with isometry group $\mathcal{G}$ acting in $\mathbb{H}^{3}$ without fixed point. The orbit space will be isometric to the identified starting simplex or double-simplex, respectively, as indicated above. The so-called Dehn-surgery, introduced by Thurston [23], deforms the ideal simplices to have special angles different from $\pi / 3$, such that a starting polyhedron $\tilde{\mathcal{P}}$ will be no more fundamental domain of the induced group $\mathcal{G}(\mathcal{I})$ in the original sense. However, $\mathbb{H}^{3} / \mathcal{G}$ can be compact cone manifold, especially an orbifold with rotational axis for singular points.

Of course, this cone manifold can also be presented by a compact polyhedron, then with the same volume as the starting $\tilde{\mathcal{P}}$ having ideal vertices. We shall compute these angular parameters and the volume of $\tilde{\mathcal{P}}$ by the classical method of N. I. Lobachevsky (see e.g. in [24]). All computations are carried out by Maple 5.0 on the base of the half-space model of the Bolyai-Lobachevsky hyperbolic space $\mathbb{H}^{3}$, where the ideal points at infinity are described by $\mathbb{C} \cup$ $\{\infty\}=: \mathbb{C}_{\infty}$ as complex projective line $[5,11,18,23]$. The interior point $(w, \zeta)$ of $\mathbb{H}^{3}$ over $w \in z_{1} z_{2} \subset \mathbb{C}_{\infty}$ (in a half circle) has the third coordinate $\zeta>0$ with $\left|z_{1}-w\right|\left|w-z_{2}\right|=\zeta^{2}$ (see Fig. 1b).

We do not discuss here the important connections with knot theory, e.g. $[4,10$, $23,25]$ and other algorithmic, computational method, e.g. [1-3, 6, 8, 20-22, 26].

\section{Gieseking manifold and its surgeries}

We start with the ideal simplex of $\mathbb{H}^{3}$ in the half-space model (Fig. 1), where its ideal vertices at infinity are represented by

$$
0,1, z, \infty \in \mathbb{C} \cup\{\infty\}=: \mathbb{C}_{\infty}
$$


of the complex projective line. This will be an identified ideal simplex $\tilde{\mathcal{S}}$ with face pairing mappings $\mathbf{z}_{1}$ and $\mathbf{z}_{2}$ as "horospherical glide reflections"

$$
\begin{aligned}
& \mathbf{z}_{1}: \infty z 1=:\left[z_{1}^{-1}\right] \rightarrow \infty 10=:\left[z_{1}\right], \text { i.e. } \\
& \mathbf{z}_{1}: u \mapsto \frac{\bar{u}-1}{\bar{z}-1} \text { or }(u, 1) \mapsto(\overline{u, 1})\left(\begin{array}{cc}
1 & 0 \\
-1 \bar{z}-1
\end{array}\right), \\
& \mathbf{z}_{2}: 0 z \infty=:\left[z_{2}^{-1}\right] \rightarrow 01 z=:\left[z_{2}\right], \text { i.e. } \\
& \mathbf{z}_{2}: u \mapsto \frac{\bar{u} z}{\bar{u}-\bar{z}(1-z)} \text { or }(u, 1) \mapsto(\overline{u, 1})\left(\begin{array}{l}
z 1 \\
0-\bar{z}(1-z)
\end{array}\right) .
\end{aligned}
$$

As usual (e.g. in [24]), we extend the actions of the transformations into the upper half space by half-circles and half spheres orthogonally to the boundary plane, represented by $\mathbb{C}_{\infty}$. Circles and spheres through the infinity $\infty$ will be orthogonal half lines and half planes, respectively. Thus we can describe the lines and the planes of the model space of $\mathbb{H}^{3}$, moreover its congruence transformations.

Going round e.g. the edge $\overrightarrow{\infty z}$ from the starting identity 1 simplex, we meet first the face $z_{1}^{-1}$, then follows, on the other side, the image face $\left[z_{1}\right]^{\mathbf{z}_{1}^{-1}}$ $\left(=z_{1}^{-1}\right)$ at the edge $(\infty 1)^{\mathbf{z}_{1}^{-1}}$ in the $\mathbf{z}_{1}^{-1}$-image simplex. Then the image face $\left[z_{1}\right]^{\mathbf{z}_{1}^{-1}}$ and, on the other side, the face $\left[z_{1}\right]^{\mathbf{z}_{1}^{-1} \mathbf{z}_{1}^{-1}}\left(=\left[z_{1}^{-1}\right]^{\mathbf{z}_{1}^{-1}}\right)$ come at edge $(\infty 0)^{\mathbf{z}_{1}^{-1}}$ in the $\mathbf{z}_{1}^{-1} \mathbf{z}_{1}^{-1}$-image simplex. Then we meet the image face $\left[z_{2}^{-1}\right]_{1}^{-1} \mathbf{z}_{1}^{-1}$ and, on the other side, the image simplex $\mathbf{z}_{2}^{-1} \mathbf{z}_{1}^{-1} \mathbf{z}_{1}^{-1}$ by the conjugate mapping $\mathbf{z}_{1} \mathbf{z}_{1} \mathbf{z}_{2}^{-1} \mathbf{z}_{1}^{-1} \mathbf{z}_{1}^{-1}$ of $\mathbf{z}_{2}^{-1}$. Thus [12], we obtain the cycle transformation $\mathbf{z}_{2} \mathbf{z}_{1} \mathbf{z}_{2}^{-1} \mathbf{z}_{2}^{-1} \mathbf{z}_{1}^{-1} \mathbf{z}_{1}^{-1}$ and we prescribe the trivial rotation order $\nu=1$ for the unique edge class containing 6 edges. Finally we get the defining cycle relation

$$
\mathbf{z}_{1} \mathbf{z}_{1} \mathbf{z}_{2} \mathbf{z}_{2} \mathbf{z}_{1}^{-1} \mathbf{z}_{2}^{-1}=\mathbf{1}
$$

for the fundamental group of the Gieseking manifold in equivalent form. All these are in conformity with the fact that the dihedral angles of a regular ideal simplex are $\pi / 3,6 \cdot(\pi / 3)=2 \pi$ will guarantee ball-like neighbourhood of any point at simplex edges. However, the relation (2.3) with (2.2) - by careful computations - leads to equivalent equation

$$
|z-1|^{2}=|z|
$$

with more general ideal simplex, not necessarily the regular one.

Now, we turn to the ideal vertex class forming a cusp (Figs. 1, 2). This will be represented by gluing; corresponding to images of the 4 vertex domains to that of $\infty$. The side face pairing of $\tilde{\mathcal{S}}$ induces the pairing of the sides of a 2-dimensional polygon, denoted by $\tilde{s}$ in Figs. 1 and 2, say, on a horosphere centred in $\infty$. This is represented in our half-space model by a Euclidean plane parallel to the absolute, and it can also be described on the absolute by $\mathbb{C}_{\infty}$.

Topologically, the polygon $\tilde{s}$ is a Klein-bottle with fundamental group equivariant to the Euclidean crystallographic plane group 4. pg. This group, as the stabilizer $\mathcal{G}_{\infty}$ of $\infty$, is determined by the starting group $\mathcal{G}\left(\mathbf{z}_{1}, \mathbf{z}_{2}\right)$ in formulae 
(2.2). Figure 2 exactly (for $k=3$ and $k=2$, respectively) shows the more general situation that $\mathcal{G}_{\infty}$ is generated by pairing of $s$ :

$\mathbf{z}_{1}:\left[z_{1}^{-1}\right] \rightarrow\left[z_{1}\right]$ "glide reflection" as before; then

$$
\begin{aligned}
& \mathbf{p}:\left[z_{1}^{-1}\right]^{*}:=\left[z_{1}^{-1}\right]^{\mathbf{z}_{2}^{-1}} \rightarrow\left[z_{1}\right]^{*}:=\left[z_{1}\right]^{\mathbf{z}_{2}^{-1} \mathbf{z}_{2}^{-1}} \\
& \text { i.e. } \mathbf{p}=\mathbf{z}_{2} \mathbf{z}_{1} \mathbf{z}_{2}^{-1} \mathbf{z}_{2}^{-1}=\mathbf{z}_{1} \mathbf{z}_{1}: u \rightarrow(u-z) /|z|
\end{aligned}
$$

a "translation" as a central similarity in $\mathbb{C}_{\infty}$,

$$
\begin{aligned}
& \mathbf{z}_{2}^{*}:\left[z_{2}^{-1}\right]^{*}:=\left[z_{2}^{-1}\right]^{\mathbf{z}_{1}^{-1} \mathbf{z}_{2}^{-1} \mathbf{z}_{2}^{-1}} \rightarrow\left[z_{2}\right]^{*}:=\left[z_{2}\right]^{\mathbf{z}_{1}^{-1} \mathbf{z}_{2}^{-1} \mathbf{z}_{2}^{-1}}, \\
& \text { i.e. } \mathbf{z}_{2}^{*}=\mathbf{z}_{2} \mathbf{z}_{2} \mathbf{z}_{1} \mathbf{z}_{2} \mathbf{z}_{1}^{-1} \mathbf{z}_{2}^{-1} \mathbf{z}_{2}^{-1} \text { a "glide reflection" }
\end{aligned}
$$

again, it is conjugated to $\mathbf{z}_{2}$. We see that $\mathbf{p}$ is a "translation", it is $\mathbf{z}_{2}^{-1}$ conjugated to $\mathbf{z}_{1} \mathbf{z}_{2}^{-1}$. This group $\mathcal{G}_{\infty}$ is 4 . pg itself (on the Euclidean plane represented by $\mathbb{C}_{\infty}$ ) if $z=\frac{1}{2}+i \frac{\sqrt{3}}{2}$. We have obtained the Gieseking manifold with one cusp. Other $z$, as a complex parameter, makes the stabilizer $\mathcal{G}_{\infty}$ to a conformal group with fixed points

$$
\infty \text { and } v:=\frac{z}{1-|z|} \text {. }
$$

This line $v \infty$ will not be covered by the $\mathcal{G}_{\infty}$-images of the simplex $\tilde{\mathcal{S}}$ in $\mathbb{H}^{3}$. In the model half-space the translations of $\mathcal{G}_{\infty}$ in (2.5) by (2.2) will be similarities of $\mathbb{C}_{\infty}$ with fixed points $v, \infty$. E.g. $\mathbf{z}_{1}$ in (2.2) and $\mathbf{z}_{2}$ in (2.5) are similarityreflections indicated in Figs. 2, 3, 4 and 5. The simple ratio on 01 is $u:=$ $1 /(1+|z-1|)$. For $\mathbf{z}_{2}^{*}$ in $(2.5)$ we can write by $(2.2)$

$$
\begin{aligned}
& \mathbf{z}_{2}^{*}:(u, 1) \rightarrow(\overline{u, 1})\left(\begin{array}{ll}
1 & 1 \\
0 & |1-z|^{2}
\end{array}\right)\left(\begin{array}{cc}
1 & 0 \\
-1 & \bar{z}-1
\end{array}\right) \\
& \cdot\left(\begin{array}{ll}
\bar{z} 1 & \\
0-z(1-\bar{z})
\end{array}\right)\left(\begin{array}{cc}
z-1 & 0 \\
1 & 1
\end{array}\right)\left(\begin{array}{cc}
|1-z|^{2}-1 \\
0 & 1
\end{array}\right) \\
& \stackrel{(2.4)}{=}(\overline{u, 1})\left(\begin{array}{ll}
z(1-\bar{z})^{2} & 0 \\
|z|^{2}\{|z|(\bar{z}-1)-(|z|+1)\}-\bar{z}(1-z)
\end{array}\right) .
\end{aligned}
$$

Now, we turn to the critical, so-called surgery transform $\mathbf{z}_{1} \mathbf{z}_{2}^{*}$. By the tricky use of (2.4), as

$$
|z|=|z-1|^{2}=(z-1)(\bar{z}-1)=|z|^{2}-z-\bar{z}+1,
$$

we obtain

$$
\mathbf{z}_{1} \mathbf{z}_{2}^{*}:(u, 1) \rightarrow(u, 1)\left(\begin{array}{ll}
z(1-\bar{z})^{2} & 0 \\
\{|z|+1\}\left[|z|^{2}-z^{2}\right] \bar{z}(1-z)^{2}
\end{array}\right),
$$

fixing $\infty$ and $v$, of course. We see by (2.4) that $\mathbf{z}_{1} \mathbf{z}_{2}^{*}$ describes a rotation of the model half-space about the line $\infty v$ with angle

$$
\phi:=\arg \left[\frac{z(1-\bar{z})^{2}}{\bar{z}(1-z)^{2}}\right]=2 \arg z-4 \arg (1-z)(\bmod 2 \pi),
$$

i.e. $\phi=2 \cdot \alpha_{1}+4 \cdot \alpha_{3}(\bmod 2 \pi)$. 

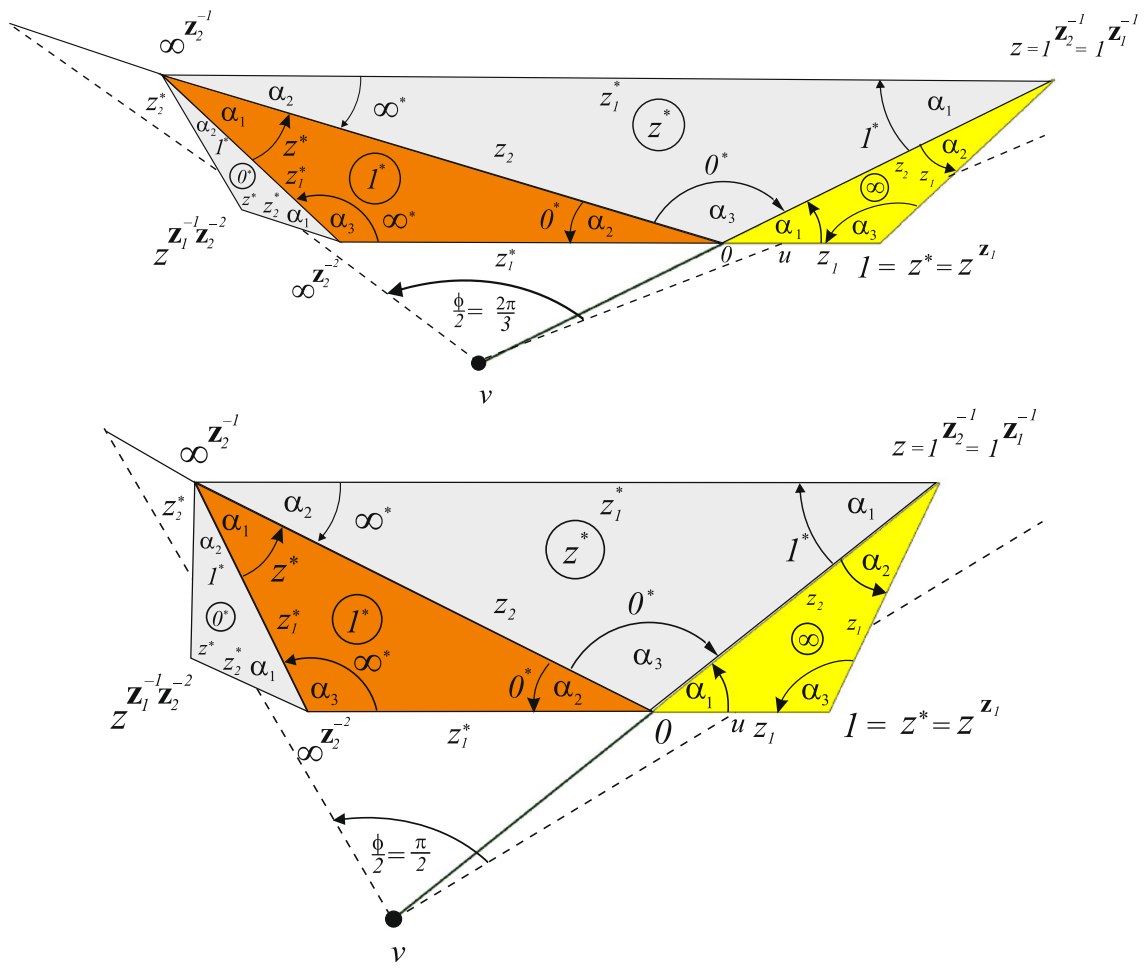

Figure 2 a The topological Klein bottle group 4. pg in $\infty$ of $\mathbb{C}_{\infty}$ glued by fundamental domain $\mathcal{S} ; \mathbf{z}_{1} \mathbf{z}_{2}^{*}$ is a rotation through $-2 \pi / k=2 \pi(k-1) / k(\bmod 2 \pi)$ for $k=3$; b The orbifold for $k=2$

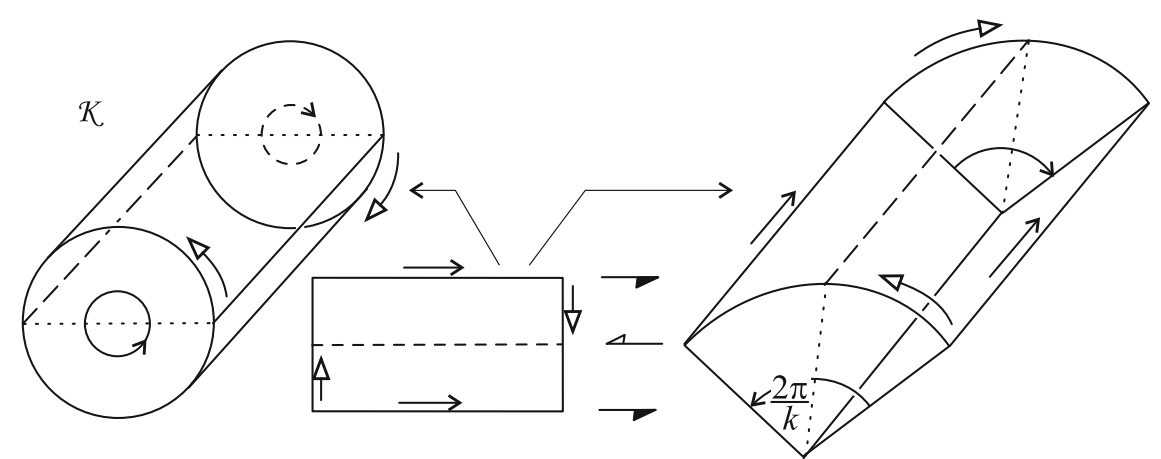

FiguRE 3 Klein bottle solids for the later manifold or cone manifold (orbifold if the cone angle is $2 \pi / k$ )

If we require the stabilizer $\mathcal{G}_{\infty}$ to act discontinuously on the model half-space, then this angle necessarily will be $\pm 2 \pi / k(\bmod 2 \pi)$, through the unit root 
parameter $\kappa=e^{ \pm i 2 \pi / k}$ i.e.

$$
\begin{aligned}
\frac{z}{(1-z)^{2}}= & \pm e^{ \pm i \pi / k}= \pm \sqrt{\kappa}, \text { with } \\
& 1<|z-1|<|z|, \text { or }|z|<|z-1|<1 \text {; and } \operatorname{Im} z>0, \quad k=2,3, \ldots
\end{aligned}
$$

can be assumed. Then $k$ is the periodicity of the rotation $\mathbf{z}_{1} \mathbf{z}_{2}^{*}$ and we get 4 root series for the fundamental simplices, called Gies.1-Gies.4. The first one is chosen

$$
z=1+\frac{1}{2} e^{i \pi / k}\left(1+\sqrt{1+4 e^{-i \pi / k}}\right), \quad k=2,3, \ldots
$$

Remark 2.1. In more general, we can write $\pm 2 \pi \cdot l / k, l=1,2, \ldots, k-1$ over (2.9) and later $\pm \pi \cdot l / k$, respectively by parameter $\kappa=e^{ \pm i 2 \pi \cdot l / k}$, in the following 4 root series for $z$. Then we obtain multiple tiling as well, and the tile itself realizes so-called cone manifold as Fig. 3a shows this phenomenon for $k=$ $3, l=2$, surprisingly a little bit. See our further Figs. 5, 6, and imagine the other cases for $(k, l)$.

All data can be computed from (2.10), especially the face angles of $\tilde{\mathcal{S}}$, equal at the opposite edges (Figs. 1a, 2b)

$$
\begin{aligned}
& \left\{\frac{\overline{\infty 0}}{z 1}\right\}: \alpha_{1}=\arg z ; \quad\left\{\frac{\overline{\infty z}}{\overline{10}}\right\}: \alpha_{2}=\arg \frac{z-1}{z} ; \\
& \left\{\frac{\overline{\infty 1}}{z 0}\right\}: \alpha_{3}=\arg \frac{1}{1-z} .
\end{aligned}
$$

However, the computer gives more guarantees. In Tables 1 and 2 we have computed by MAPLE the volume of $\tilde{\mathcal{S}}$ as well for some values of $k$. We know [24] that the Lobachevsky function

$$
\Lambda(x)=-\int_{0}^{x} \ln |2 \sin \xi| d \xi \quad \text { with } \quad \operatorname{Vol} \tilde{\mathcal{S}}=\Lambda\left(\alpha_{1}\right)+\Lambda\left(\alpha_{2}\right)+\Lambda\left(\alpha_{3}\right)
$$

provides the volume of the ideal simplex with the above angles. The formal monodromy group $\mathcal{G}\left(\mathbf{z}_{1}, \mathbf{z}_{2}, k\right)$ above has a unified "presentation"

$$
\mathcal{G}(k)=\left(\mathbf{z}_{1}, \mathbf{z}_{2}-\mathbf{1}=\mathbf{z}_{1} \mathbf{z}_{1} \mathbf{z}_{2} \mathbf{z}_{2} \mathbf{z}_{1}^{-1} \mathbf{z}_{2}^{-1}=\left(\mathbf{z}_{1} \mathbf{z}_{2} \mathbf{z}_{2} \mathbf{z}_{1} \mathbf{z}_{2} \mathbf{z}_{1}^{-1} \mathbf{z}_{2}^{-2}\right)_{k-1}^{k}\right) .
$$

For $k=2,3, \ldots$ we sketchily indicate by Figs. 1b, 2, 3 and 4 how to construct a compact fundamental domain $\tilde{\mathcal{F}}_{\mathcal{G}(k)}$ (in Fig. 4) by deforming an ideal vertex domain to a compact one. We introduce an edge $(e)$ on the line $\infty v$ and its $\mathbf{z}_{1}$-image with

$$
(e)=\left(E E^{\prime}\right), \quad e^{\mathbf{z}_{1}}=e^{\mathbf{z}_{2}^{*-1}}=\left(E^{\prime} E^{\prime \prime}\right), \quad\left(E E^{\prime}\right) \cap\left(E^{\prime} E^{\prime \prime}\right)=\emptyset .
$$




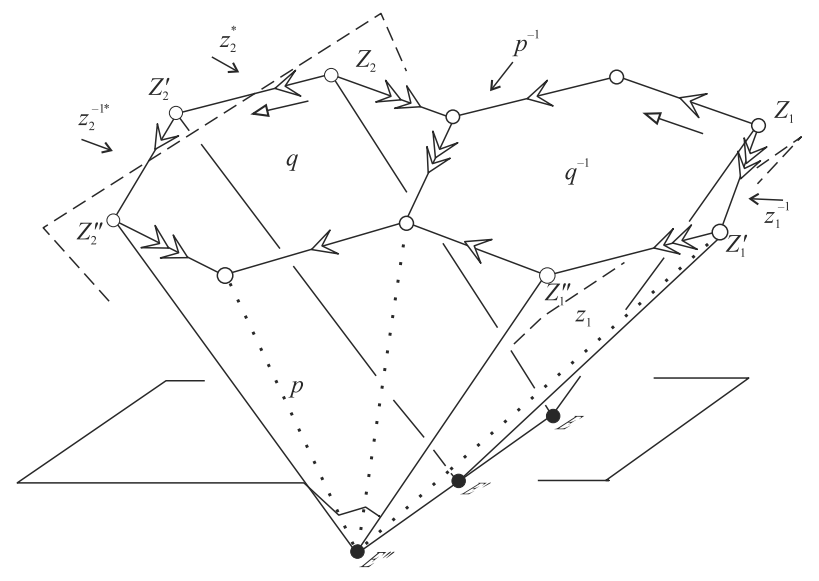

Figure 4 A sketchy combinatorial (PL, i.e. piecewise linear) compact fundamental domain for $\mathcal{G}(k)$
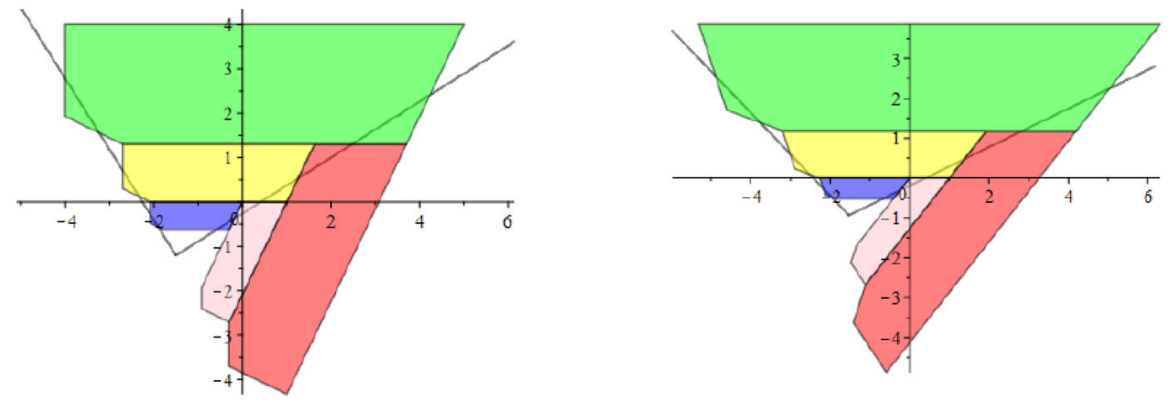

Figure 5 Gies. 1 series a $(k, l)=(2,1) ; \mathbf{b}(k, l)=(5,2)$

Then we choose a point $Z_{1}$ (e.g. the centre of the inscribed ball) in the simplex $\tilde{\mathcal{S}}=\infty 01 z$ and consider the segments

$$
\left(E Z_{1}\right), \quad\left(E Z_{1}\right)^{\mathbf{z}_{1}}=\left(E^{\prime} Z_{1}^{\prime}\right), \quad\left(E Z_{1}\right)^{\mathbf{z}_{1} \mathbf{z}_{1}}=\left(E^{\prime \prime} Z_{1}^{\prime \prime}\right) .
$$

Similarly take $Z_{2}$, as the $\mathbf{z}_{1}^{-1} \mathbf{z}_{2}^{-1} \mathbf{z}_{2}^{-1}$-image of $Z_{1}$ at the cusp gluing

$$
\left(E Z_{2}\right), \quad\left(E Z_{2}\right)^{\mathbf{z}_{2}^{*-1}}=\left(E^{\prime} Z_{2}^{\prime}\right), \quad\left(E Z_{2}\right)^{\mathbf{z}_{2}^{*-1} \mathbf{z}_{2}^{*-1}}=\left(E^{\prime \prime} Z_{2}^{\prime \prime}\right) .
$$

Then the corresponding curved (bent) surfaces $\left[q^{-1}\right]$ and its $\mathbf{z}_{1}^{-1} \mathbf{z}_{2}^{-1} \mathbf{z}_{2}^{-1}$-image $[q]$ will be constructed, transversally to the edges of $\tilde{\mathcal{S}}$ (see Figs. 1b, 2, 3, 4 and also [12]).

Finally, in Fig. 4 we get a sketchy compact fundamental domain $\tilde{\mathcal{F}}$, with piecewise linear bent faces, equipped by a pairing $\mathcal{I}\left(\mathbf{z}_{1}, \mathbf{z}_{2}^{*}, \mathbf{p}, \mathbf{q}\right)$ and (symbolic) defining relations to the corresponding edge classes:

$$
\begin{aligned}
\mathbf{z}_{1} \mathbf{z}_{1} \mathbf{p}^{-1} & =\mathbf{z}_{2}^{*} \mathbf{z}_{2}^{*} \mathbf{p}=\mathbf{q} \mathbf{z}_{2}^{*-1} \mathbf{q}^{-1} \mathbf{p q} \mathbf{q}^{-1} \mathbf{p}^{-1}=\mathbf{z}_{1} \mathbf{q} \mathbf{q} \mathbf{p}^{-1} \mathbf{q}^{-1} \\
& =\left(\mathbf{z}_{1} \mathbf{z}_{2}^{*}\right)_{l=k-1}^{k}=\mathbf{1}
\end{aligned}
$$



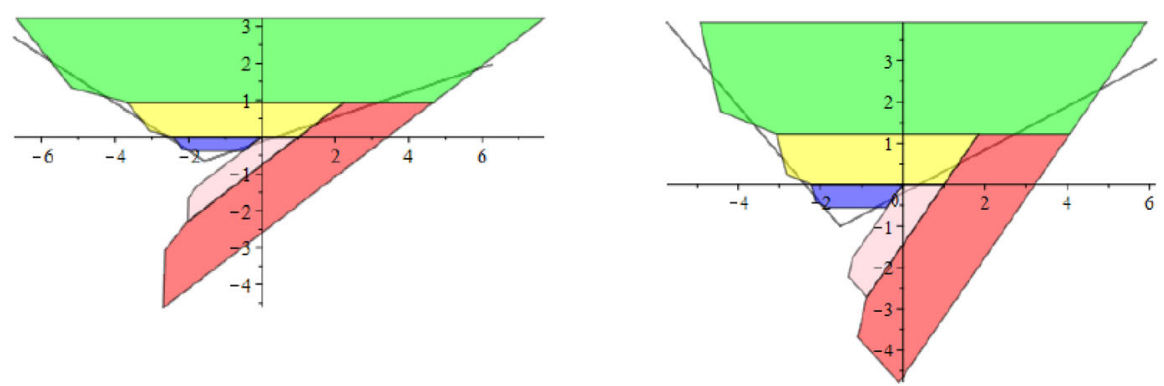

Figure 6 Gies. 1 series a $(k, l)=(7,2) ; \mathbf{b}(k, l)=(7,3)$

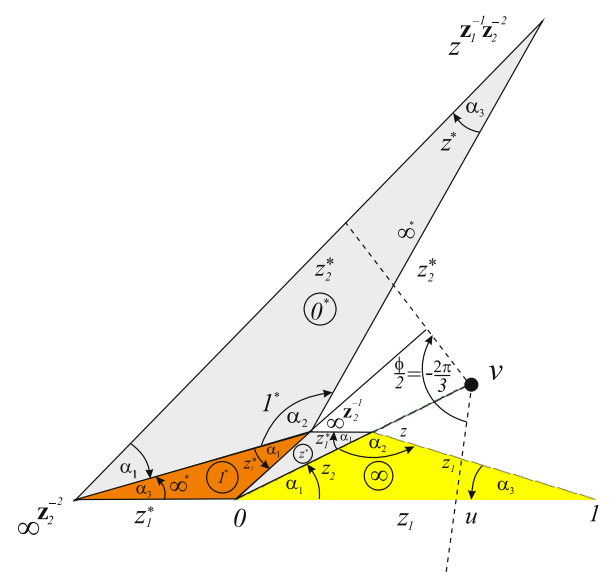

Figure 7 Gies.2 series to Tables 3 and 4 by cone manifold for $k=3, l=1$ and multiple tiling of $\mathbb{C}_{\infty}$

Of course, (2.14) equivalent to (2.13) if

$$
\mathbf{p}=\mathbf{z}_{1}^{2}, \quad \mathbf{z}_{2}^{*}=\left(\mathbf{z}_{2}^{2} \mathbf{z}_{1}\right) \mathbf{z}_{2}\left(\mathbf{z}_{1}^{-1} \mathbf{z}_{2}^{-2}\right), \quad \mathbf{q}=\mathbf{z}_{1}^{-1} \mathbf{z}_{2}^{-2} .
$$

Remark 2.2. Observe that our compactification procedure works for more general $(k, l)$ as Figs. 3 and 4 indicates. The cusp of our ideal simplex $\tilde{\mathcal{S}}$ as a Klein-bottle can be glued by a "solid Klein-bottle" $\mathcal{K}$. Then the splitting effect also occurs. The cusp of $\tilde{\mathcal{S}}$ will be cut along a Klein-bottle surface to get a boundary. Then we glue to this boundary the boundary of $\mathcal{K}$.

\subsection{The second variant of our cone manifold series}

The requirements in (2.9) provide the second root series

$$
z=1+\frac{1}{2} e^{-i \pi / k}\left(1-\sqrt{1+4 e^{i \pi / k}}\right), \quad k=2,3, \ldots
$$



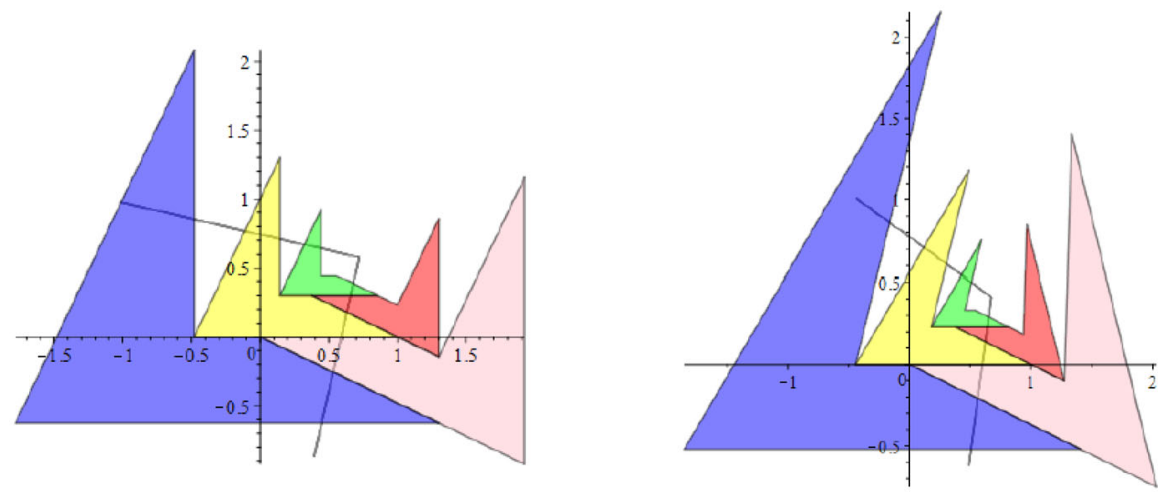

Figure 8 Gies.2 series a $(k, l)=(2,1) ; \mathbf{b}(k, l)=(5,2)$
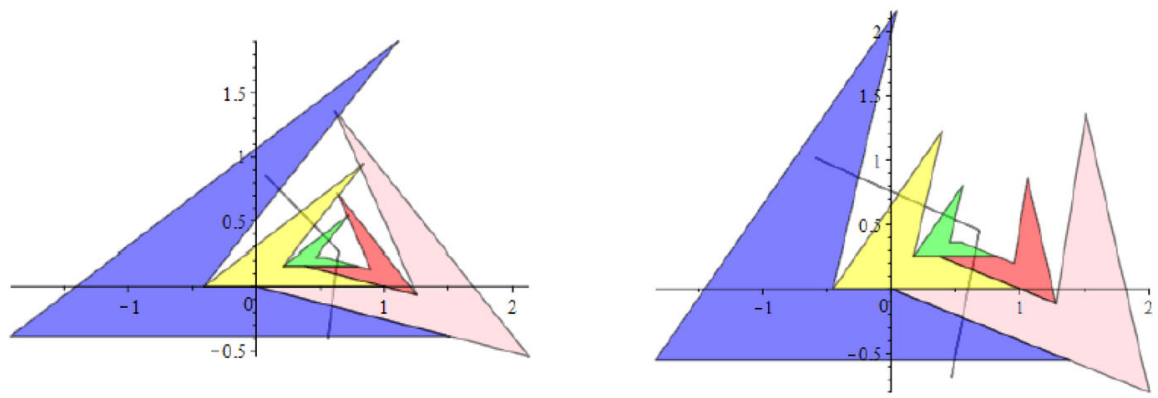

Figure 9 Gies.2 series a $(k, l)=(7,2) ; \mathbf{b}(k, l)=(7,3)$

and the cone manifold series Gies. 2 for $k>2$. Our Figs. 7, 8 and 9 and Tables 3 and 4 show these, surprisingly a little bit.

This will be geometrically equivalent to Gies.1 by the half-turn symmetry of ideal simplex: $0 \leftrightarrow \infty, 1 \leftrightarrow z$.

\subsection{Gies.3-4 tend to the regular ideal simplex manifold}

The requirements in (2.9) provide the third root series

$$
z=1-\frac{1}{2} e^{-i \pi \cdot l / k}\left(1+\sqrt{1-4 e^{i \pi \cdot l / k}}\right), \quad k=2,3, \ldots, l=1, \ldots,\lfloor k / 2\rfloor,
$$

(i.e. the lower integer part of $k / 2$ ).

and the cone manifold (orbifold) series Gies.3. Our Fig. 10 show the case $k=3$ and 9. Table 5 gives computer results. See also Figs. 11 and 12.

The fourth root series will be

$$
z=1-\frac{1}{2} e^{i \pi \cdot l / k}\left(1-\sqrt{1-4 e^{-i \pi \cdot l / k}}\right), \quad k=2,3, \ldots, l=1, \ldots,\lfloor k / 2\rfloor
$$




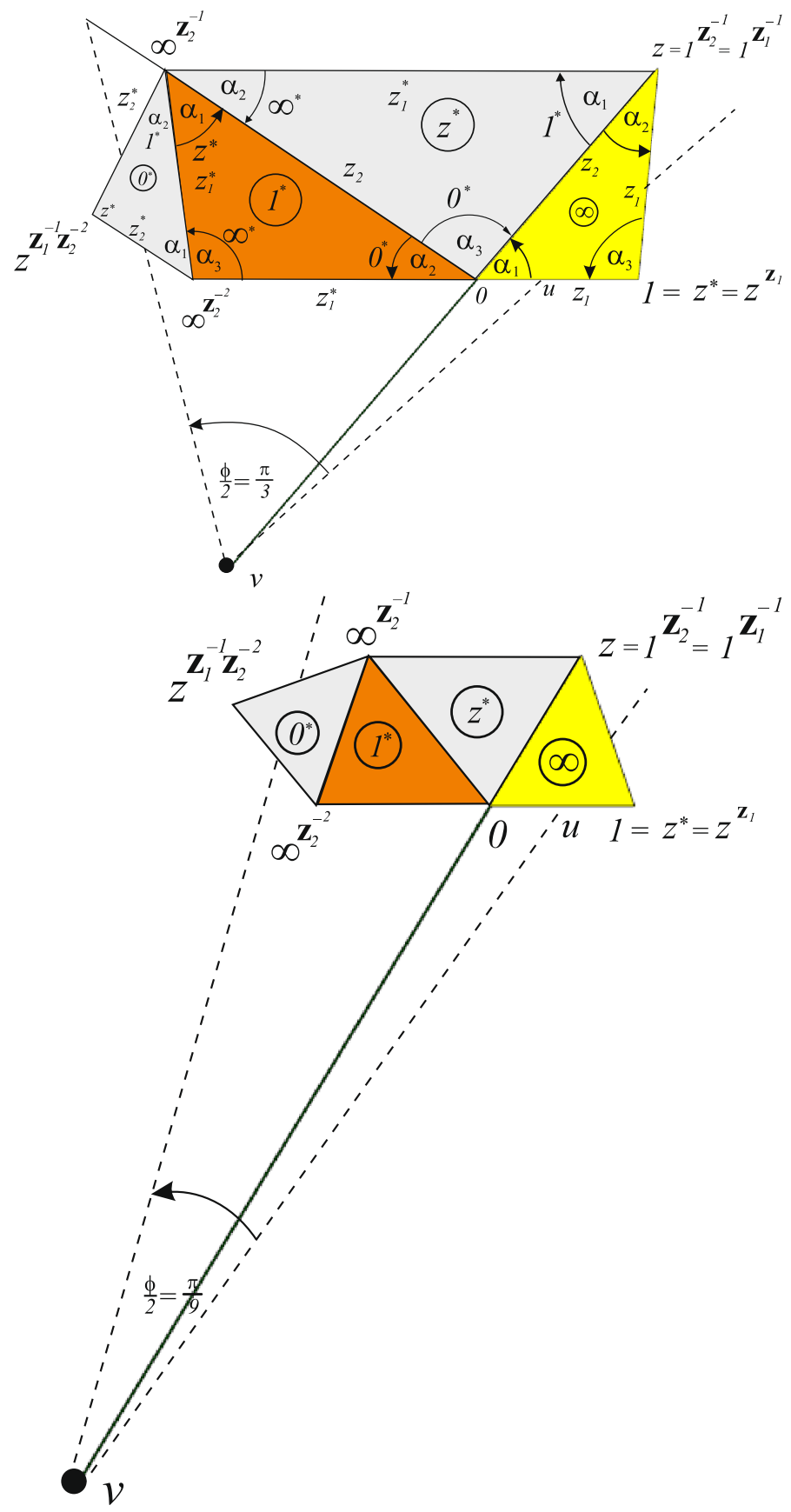

FIgURE 10 Gies. 3 series is represended by orbifolds a $k=3$; b $k=9$ 


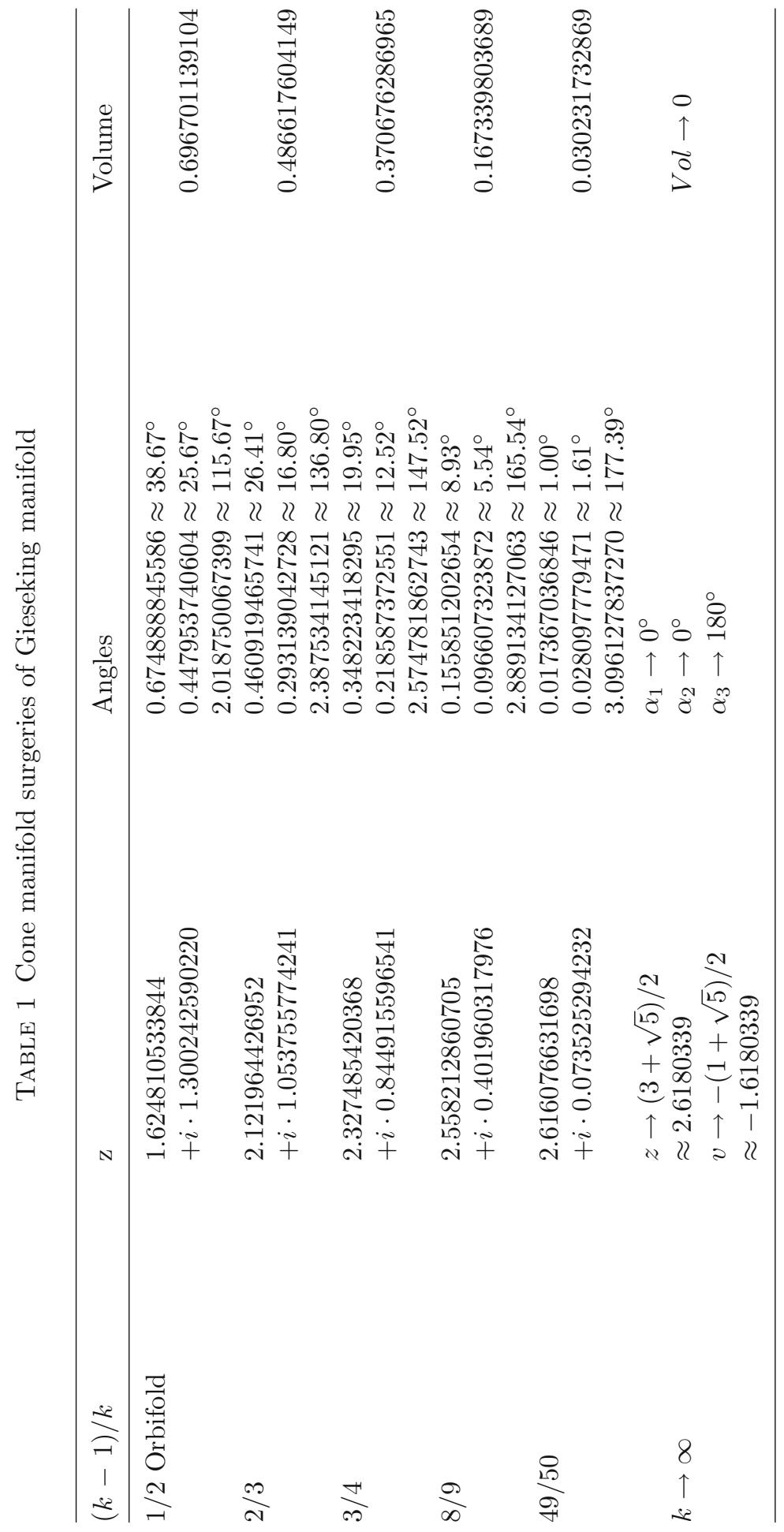




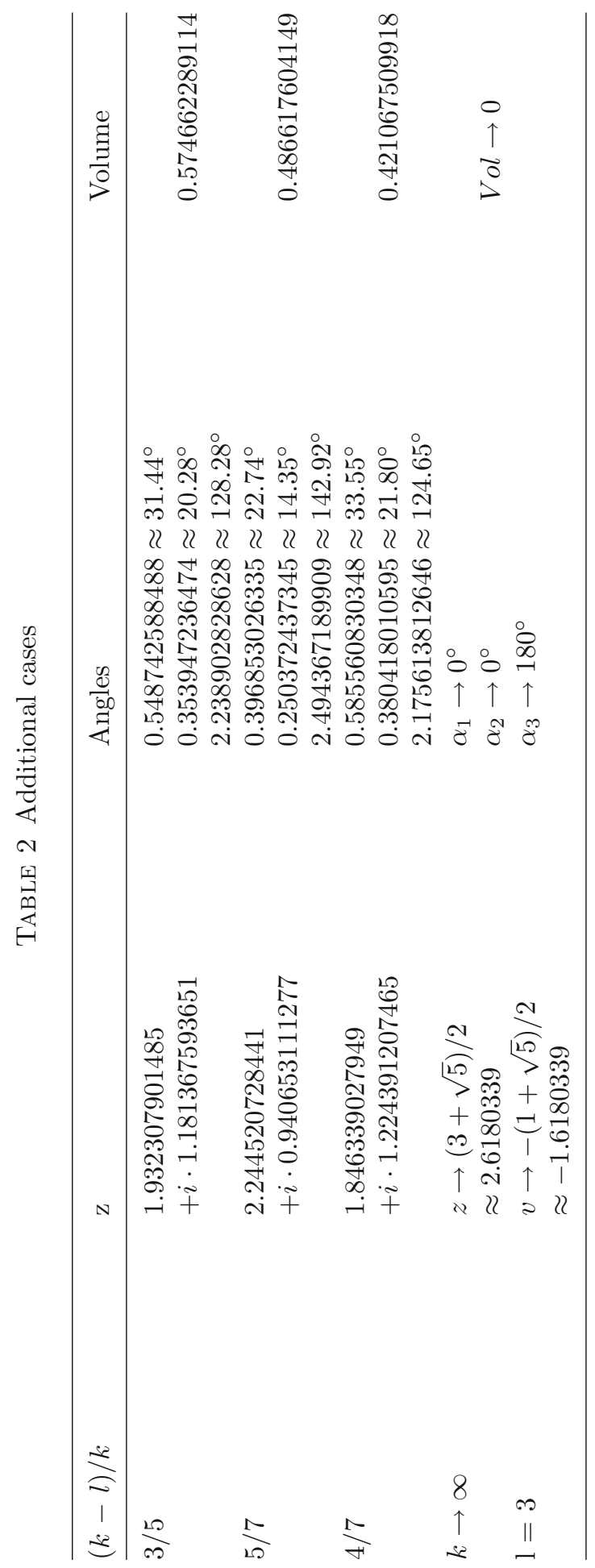




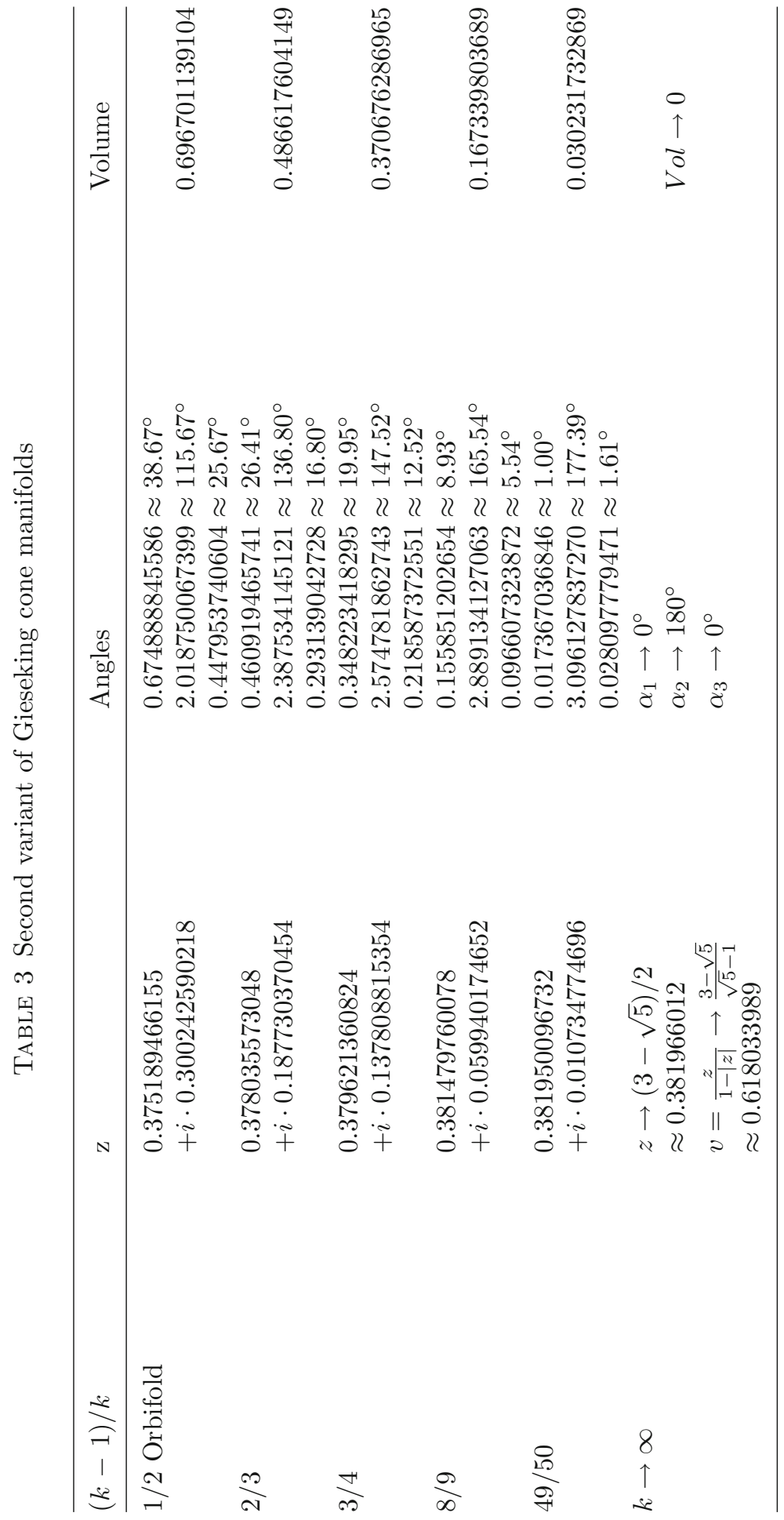




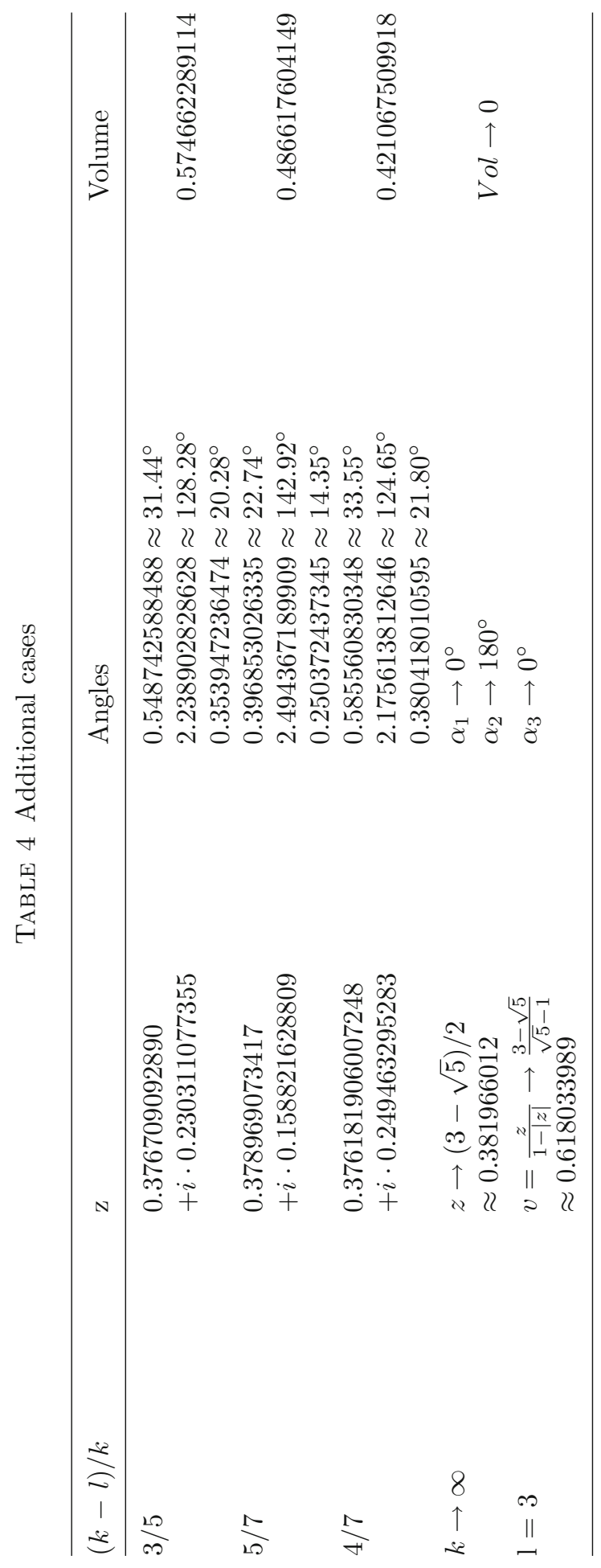




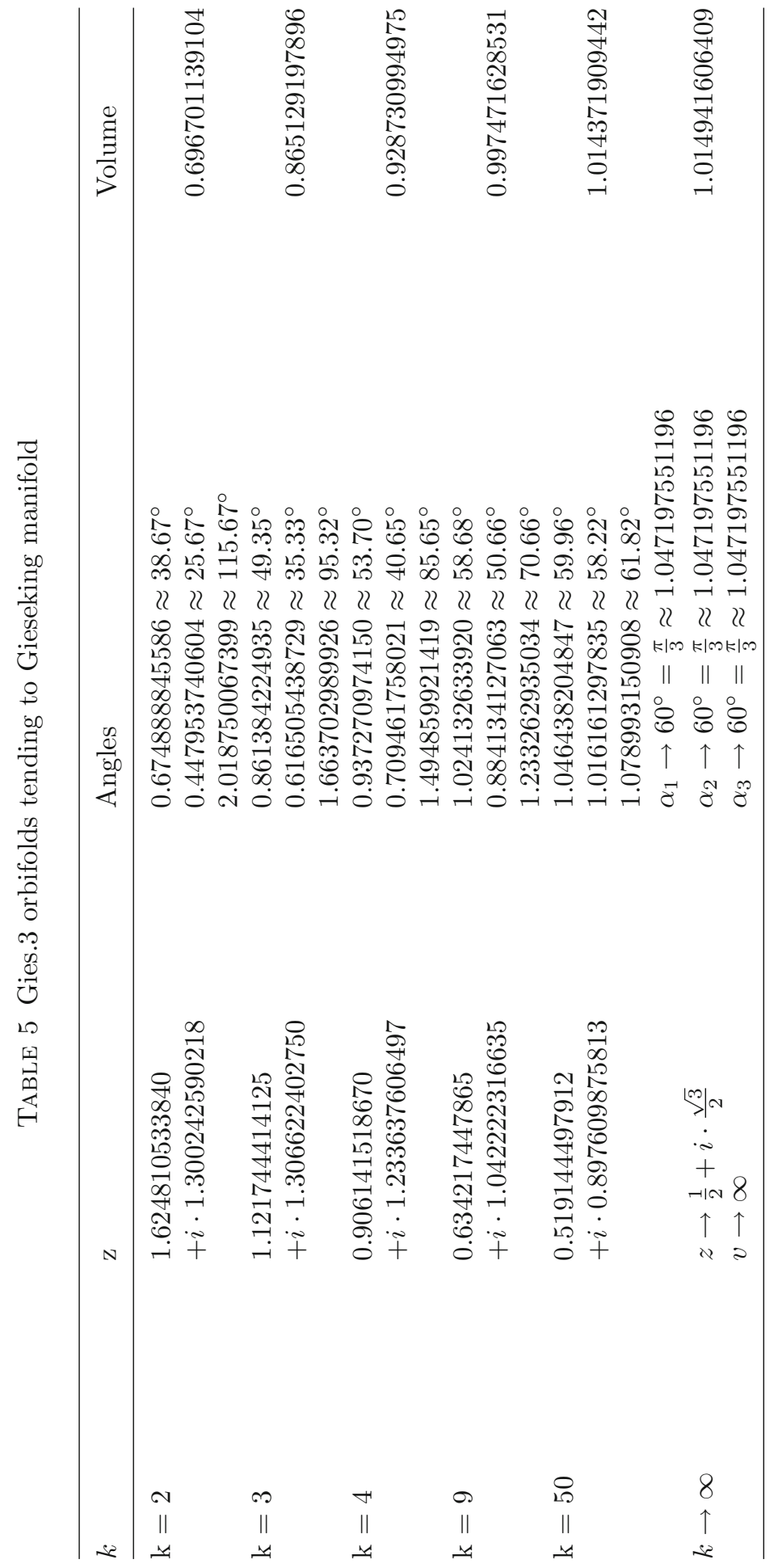




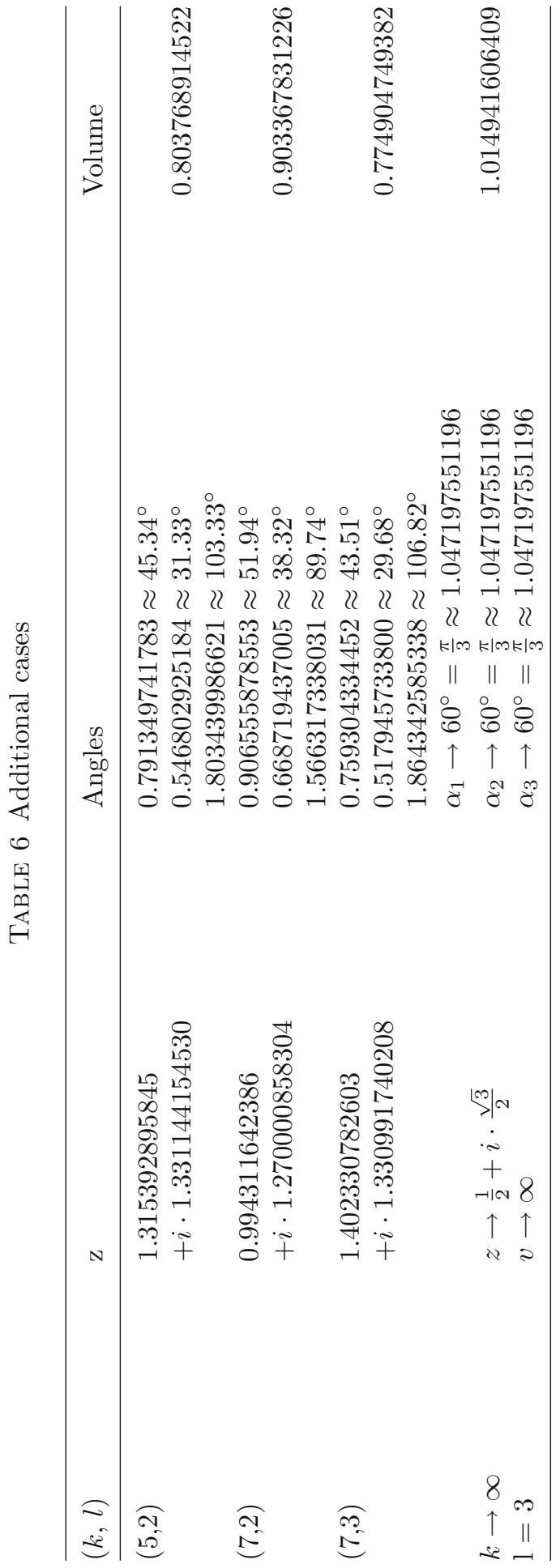



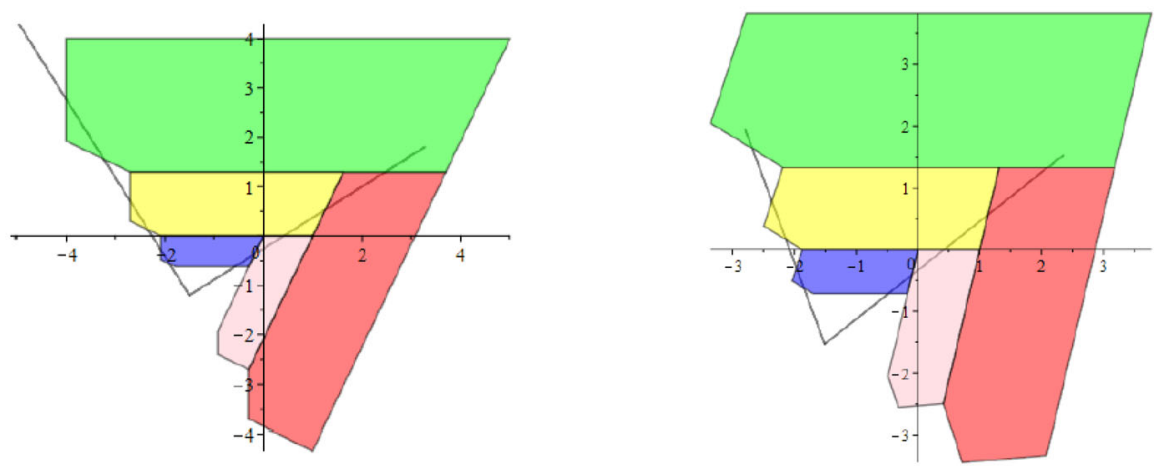

Figure 11 Gies.3 series a $(k, l)=(2,1) ; \mathbf{b}(k, l)=(5,2)$
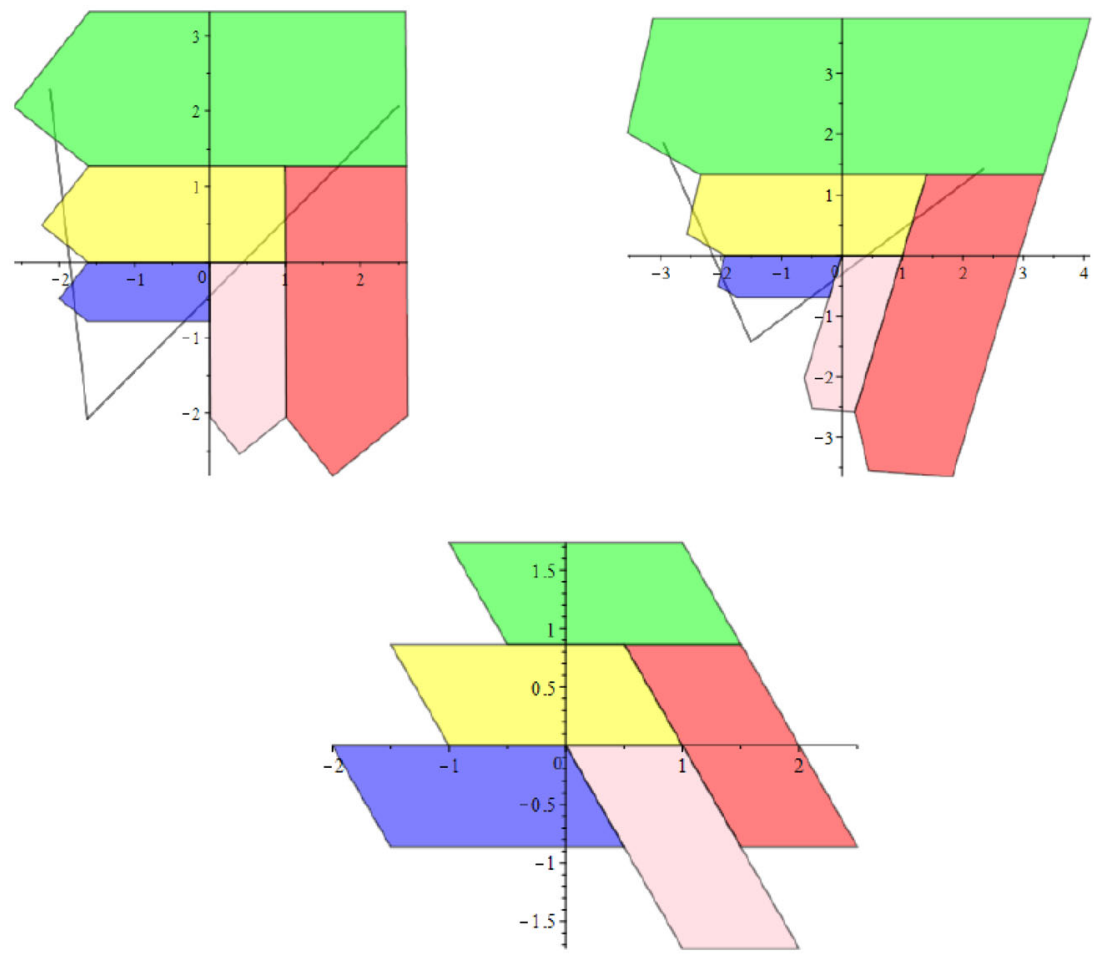

Figure 12 Gies.3 series a $(k, l)=(7,2) ; \mathbf{b}(k, l)=(7,3) ; c$. $(k, l)=(\infty, 3)$

and the cone manifold (orbifold) series Gies.4. Both last series tend to the Gieseking regular ideal simplex manifold, as J. R. Weeks predicted for us in our discussions.

We do not give here illustration to Gies.4 series, equivalent to the previous one by half-turn symmetry again. 
As we look at our Tables 1, 2, 4, 5 and 6 , and we can prove for orbifolds, $k=2$ provides the minimal volume $V=0.6967 \ldots$

Marshall and Martin [9] determined the exact lower bound $\approx 0.0390$, the next is $\approx 0.0408$ for orientable orbifolds in dimension three. Compare that with the half of the Coxeter orthoscheme $(5,3,5) \approx 0.0467$ and $(3,5,3) \approx 0.01953$, two-times less than the optimal one, but this orthoscheme has also reflection, as the authors noticed as well. In higher dimensions the problem is open, in general.

\subsection{Summary}

Now we summarize our results to Gieseking manifold.

Theorem 2.3. The surgery procedure of Gieseking manifold leads essentially to two different series for $(k, l), l=1,2, \ldots, k-1$. For rotation parameter $k=2$ we get an orbifold. For $k>2$ the surgery yields compact nonorientable hyperbolic cone manifolds and multiple tilings in the first case Gies.1-2, with underlying Gieseking manifold before, where a closed geodesic line exists with cone angle $2 \pi(k-l) / k$. This can be realized by a deformed ideal simplex $\mathcal{S}(k, l)$ with complex parameter $z(k, l)$ by Sect. 2.1 that uniquely determines all metric data in our figures and tables. The volume of $\mathcal{S}(k, l)$ tends to 0 if $k \rightarrow \infty$ for fixed $l$.

Theorem 2.4. The second series in cases Gies.3-4 (Sect. 2.1) leads to orbifolds also for $k \geq 2, l=1$ tending to the original Gieseking manifold if $k$ goes to infinity. Moreover we obtain cone manifolds and multiple tilings for other fixed $l$.

Remark 2.5. The orientable double cover of Gieseking manifold, known as Thurston manifold (or the complement of the figure-eight-knot) has a "manifold surgery" of volume $0.9813688289 \ldots$, which is known as second minimal one. But the above construction leads to cone manifold surgeries whose volumes tend to zero at the first series; and tend to the original manifold at the second series, respectively.

The minimal volume orientable compact manifold of Fomenko-Matveev-Weeks (with volume $0.94270736 \ldots$. . ) can also be obtained by manifold surgery, see also $[4,11]$. The occasionally possible (?) orbifold surgery is not examined yet (?).

We found in [16] the third non-orientable double-ideal-regular-simplex-manifold by computer. This has only cone manifold (and orbifold) surgery phenomena, i.e. with multiple (simple) tilings, respectively, that will be discussed and updated in the next Sect. 3. 


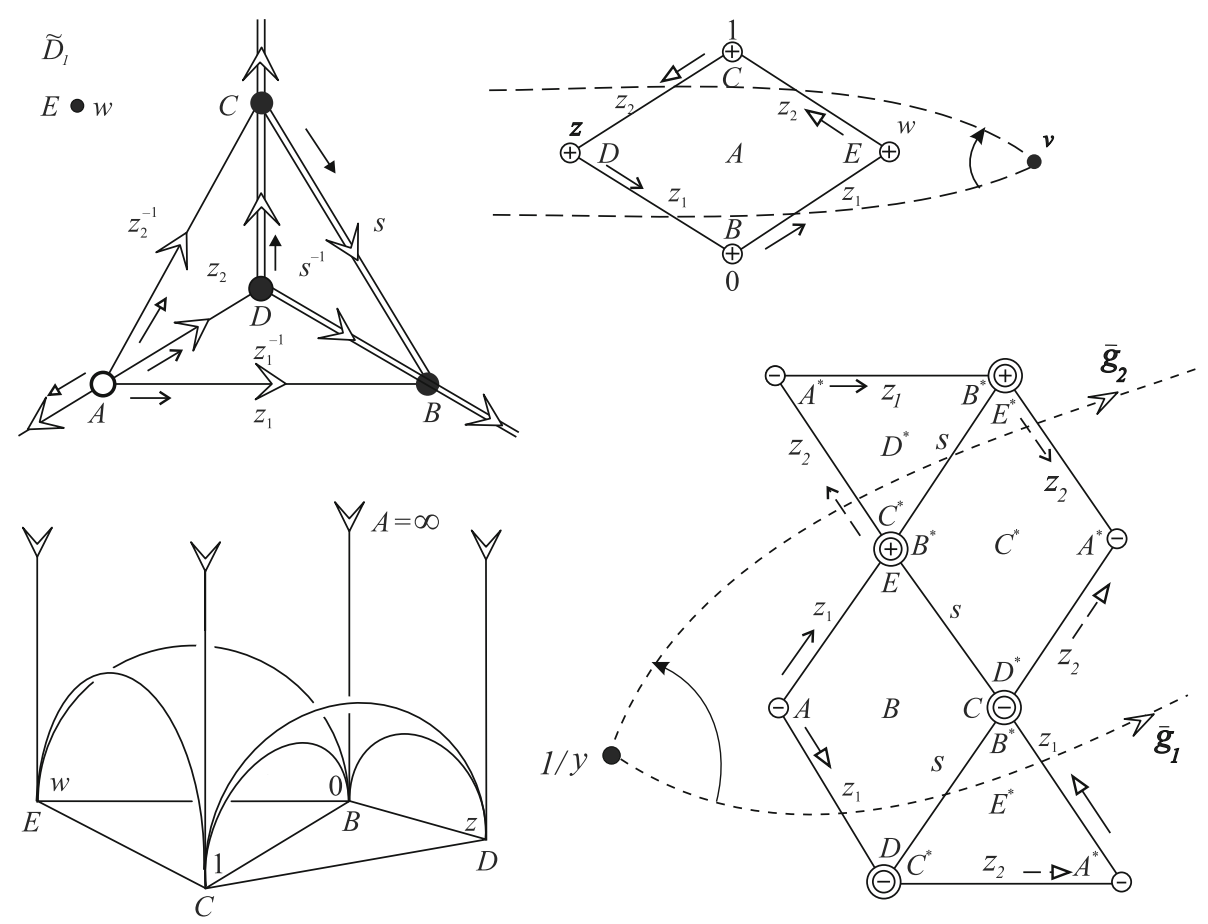

Figure 13 The non-orientable ideal double-simplex manifold $\widetilde{\mathcal{D}}_{1}$ with 2 cusps, each with Euclidean plane group 4.pg. In lower right it is described after reciprocity transform $u \rightarrow 1 / u$, $(A(\infty) \rightarrow 0, B(0) \rightarrow \infty, C(1) \rightarrow 1$, etc. $)$

\section{Double-simplex tilings; manifolds, cone mainfolds and orbifolds to non-orientable manifold $\widetilde{\mathcal{D}}_{1}$ in $\mathbb{H}^{3}$}

As we mentioned in the Abstract, a computer program tells us the possible combinatorial face pairings of the ideal double simplex $\mathcal{D}$ up to equivariance. The $\mathcal{I}$-paired double simplex $\mathcal{D}(\mathcal{I})$ will be denoted simply by $\widetilde{\mathcal{D}}$.

Two face pairings $\mathcal{I}_{1}$ and $\mathcal{I}_{2}$ of $\mathcal{D}$ are called equivariant if there is a face-to-face incidence preserving combinatorial bijection $\alpha$ of AutD , for which it holds

$$
\mathcal{I}_{2}=\alpha^{-1} \mathcal{I}_{1} \alpha, \quad \alpha \in \operatorname{Aut} \mathcal{D}=(+, 0,[] ;\{(2,2,3)\}) .
$$

AutD is equivariant to the finite spherical isometry group, with the above Macbeth signature (or *2, 2, 3 in J Conway's notation), it is of order 12, generated by 3 (plane) reflections.

Figures 14, 15, 16 and 17 list us the 7 nonequivariant possibly hyperbolic ideal double-simplices whose pairings allow us hyperbolic manifold structure. Namely, the two regular simplex components, each with face angles $\pi / 3$, allow fixed point free actions by $\mathcal{G}(\mathcal{I})$. The two edge classes with rotation orders 

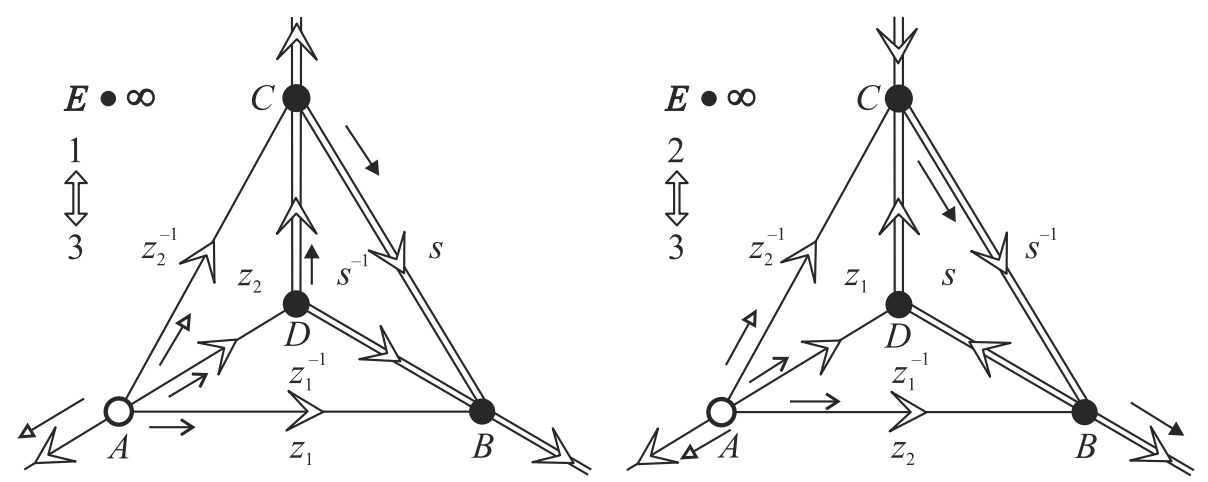

FIGURE 14 Double-simplex $\widetilde{\mathcal{D}}_{1}$, as above, and $\widetilde{\mathcal{D}}_{2}$
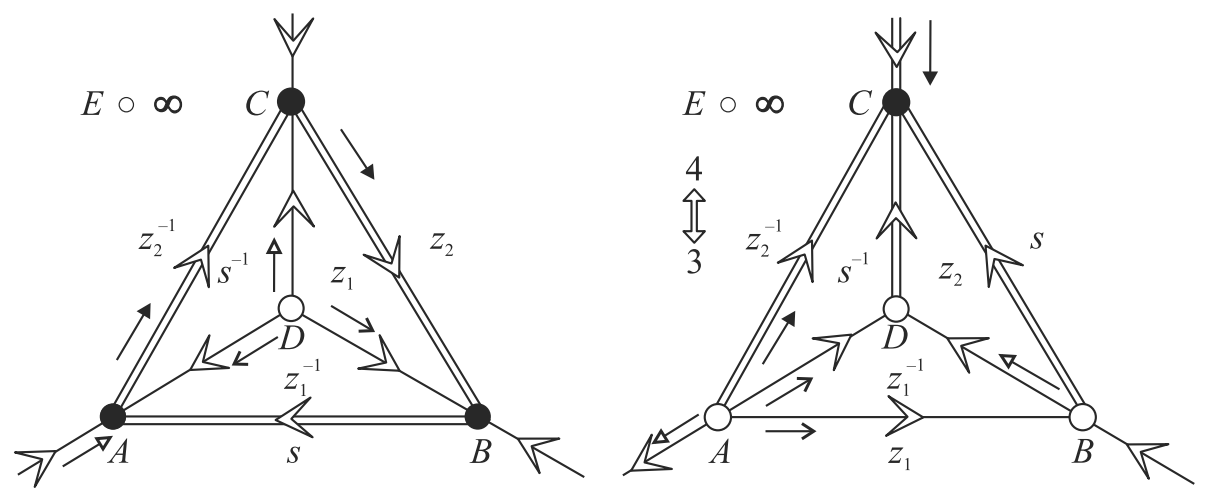

Figure 15 Double-simplex $\widetilde{\mathcal{D}}_{3}$ and $\widetilde{\mathcal{D}}_{4}$

$\nu\left(\mathfrak{e}_{1}\right)=1=\nu\left(\mathfrak{e}_{2}\right)$ also involve cusps: 1 torus in the 2 orientable cases, 2 Kleinbottles in the 5 nonorientable cases. These last ones will be represented only by $\widetilde{\mathcal{D}}_{1}$, see Figs. 13, 14, 15 and 16 and [16] for details of equivalences.

$\widetilde{\mathcal{D}}_{5}$ will be the orientable Fomenko-Matveev-Weeks manifold [5,25], whose manifold surgery led to the smallest known hyperbolic compact manifold with volume

$$
\begin{aligned}
& \operatorname{Vol}(\mathrm{FMW}) \approx 0.94270736277692772092 \quad \text { if } A(\infty), \quad B(0), \quad C(1), \\
& D(z=0.64039779245343025886+i \cdot 0.03695058574550892994) \\
& E(w=0.10601097928435957689-i \cdot 0.47247912412050095790),(3.2)
\end{aligned}
$$

see also [11] for interpretation similar to that of this paper. Here we intended for great accuracy. 

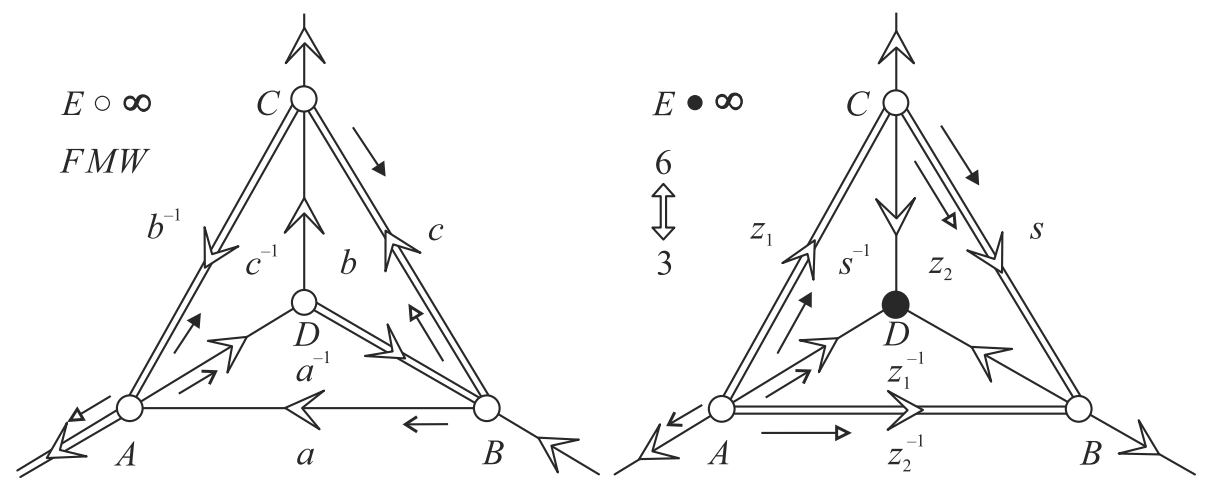

Figure $16 \widetilde{\mathcal{D}}_{5}$ as the Fomenko-Matveev-Weeks manifold. $\widetilde{\mathcal{D}}_{6}$ is equvalent to $\widetilde{\mathcal{D}}_{1}$

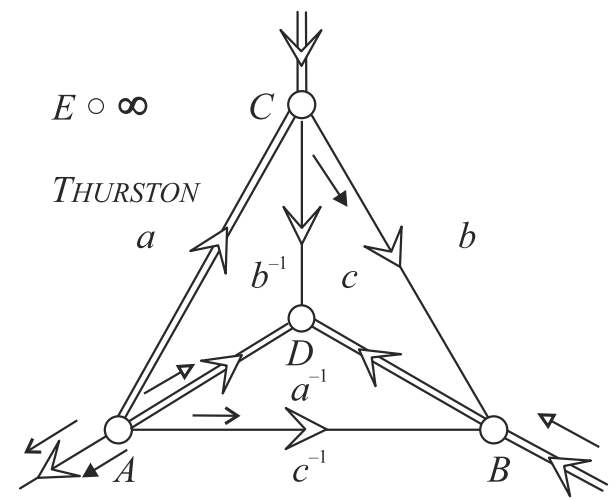

FIGURE $17 \widetilde{\mathcal{D}}_{7}$ as the Thurston manifold

$\widetilde{\mathcal{D}}_{7}$ is the Thurston manifold [23] whose manifold surgery yields a second volume known compact hyperbolic manifold with

$$
\begin{aligned}
& \operatorname{Vol}(\mathrm{T}) \approx 0.98136882889223208809 \quad \text { if } A(\infty), \quad B(0), \quad C(1), \\
& D(z=0.19043000620505709723+i \cdot 0.06928167463733572676), \\
& E(w=0.97056464193309968264-i \cdot 0.41592639156274576397) .
\end{aligned}
$$

The double-simplices $\widetilde{\mathcal{D}}_{1}, \widetilde{\mathcal{D}}_{2}, \widetilde{\mathcal{D}}_{3}, \widetilde{\mathcal{D}}_{4}, \widetilde{\mathcal{D}}_{6}$, having 2 cusp Klein-bottles will serve the same nonorientable manifold $M_{c^{2}}$, as we shall briefly indicate only by figures, but their pairings are not equivariant (see [16] for details).

In Fig. 13 we choose $\widetilde{\mathcal{D}}_{1}=M_{c^{2}}$ and carry out the analogous procedure as in Sect. 2 for the Gieseking simplex $\tilde{\mathcal{S}}$. Take the ideal vertices in the half-space model of $\mathbb{H}^{3}$, namely,

$$
A=\infty, \quad B=0, \quad C=1, \quad D=z, \quad E=w \quad \text { in } \quad \mathbb{C}_{\infty} .
$$




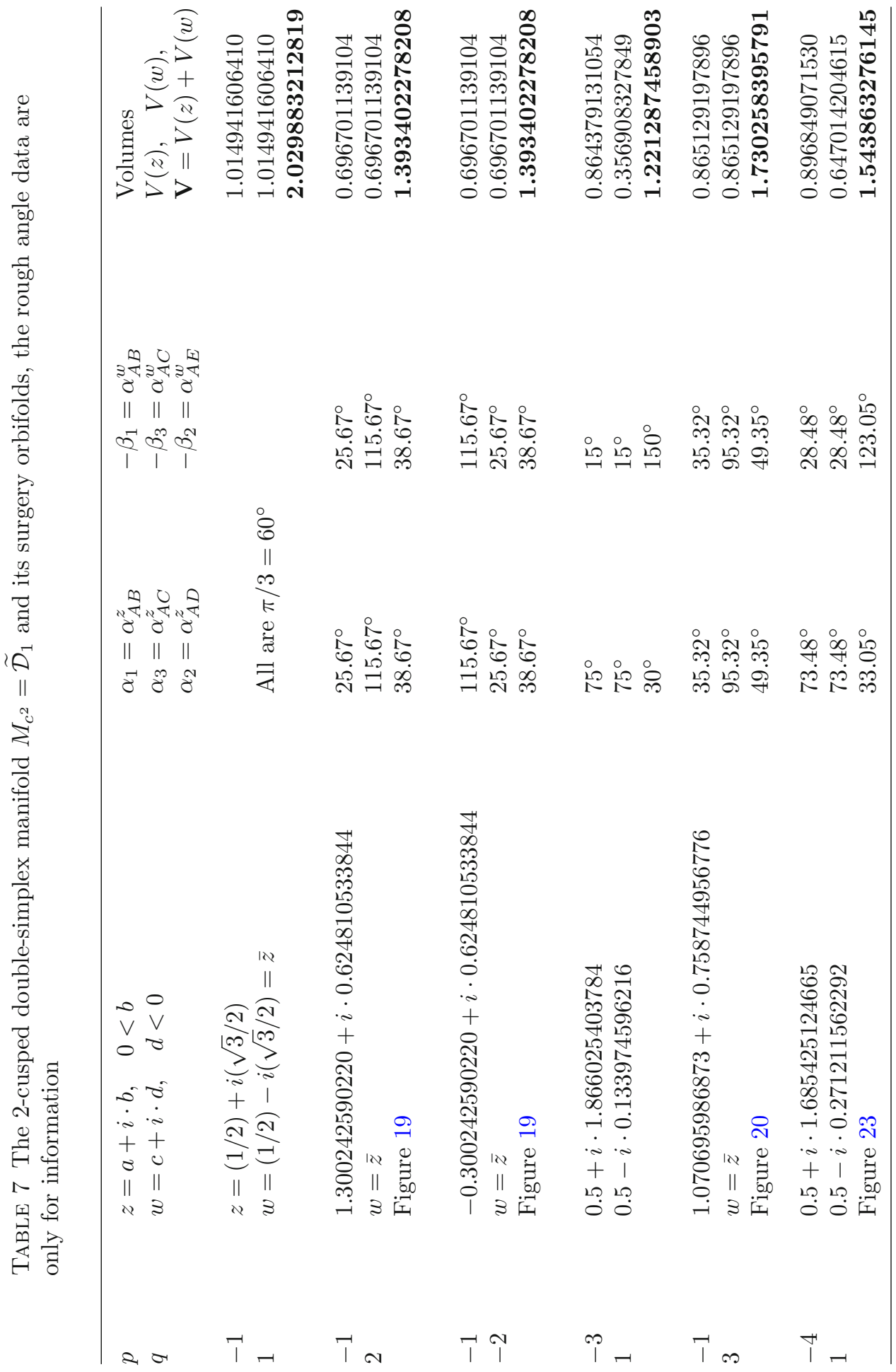




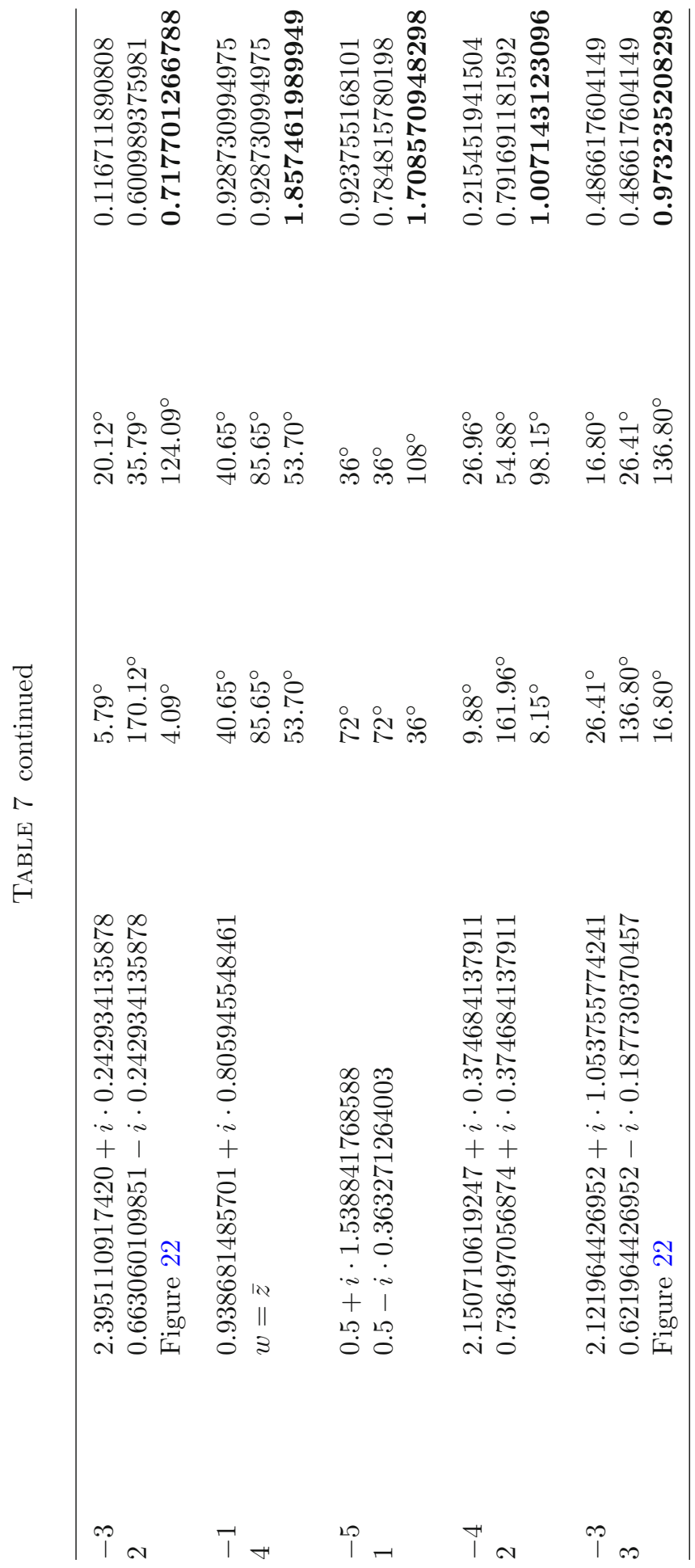




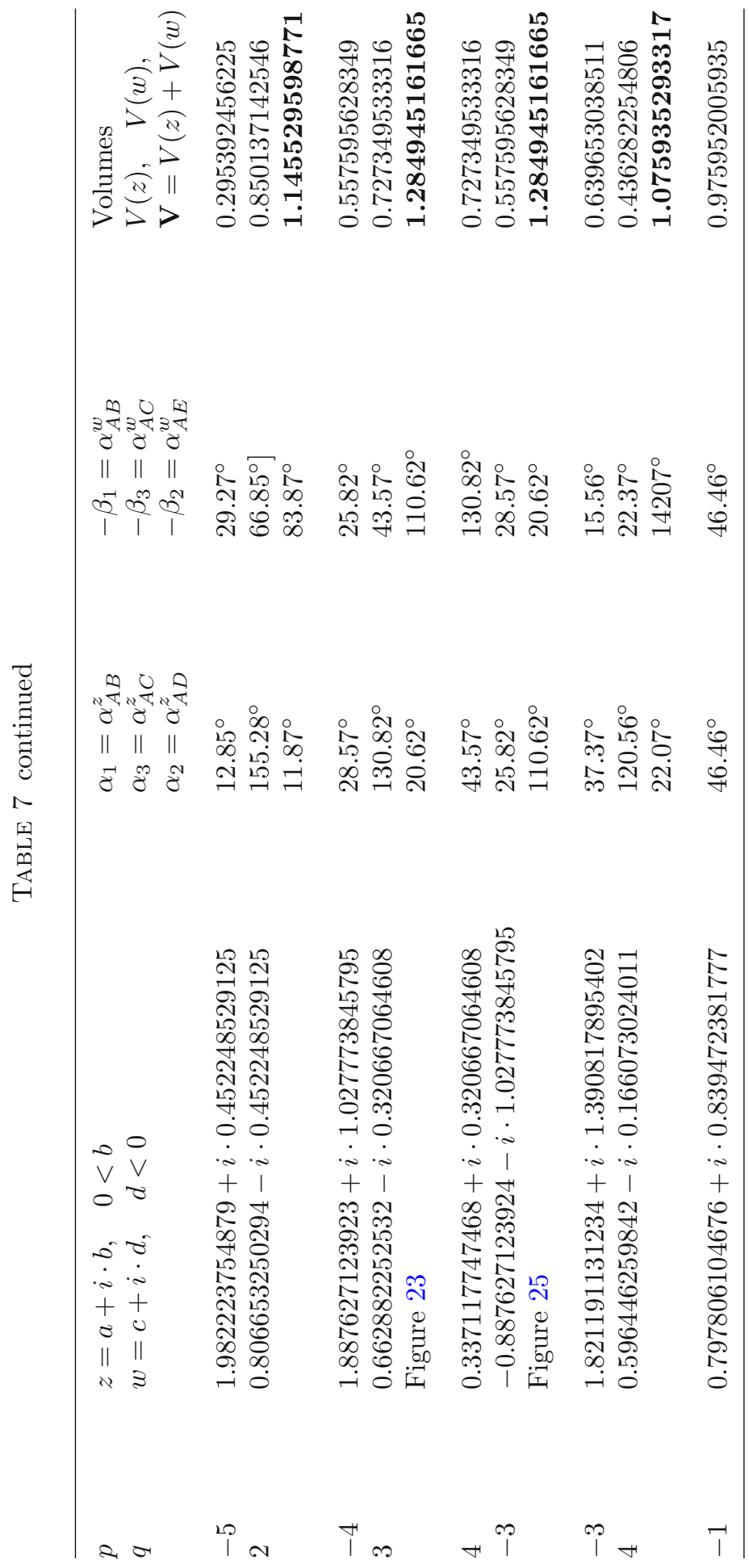




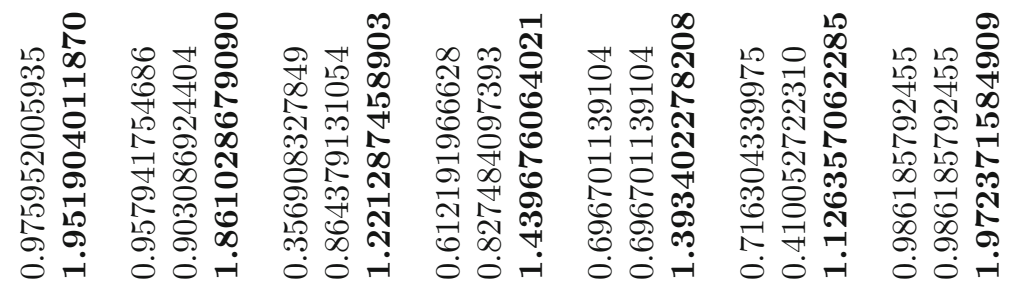

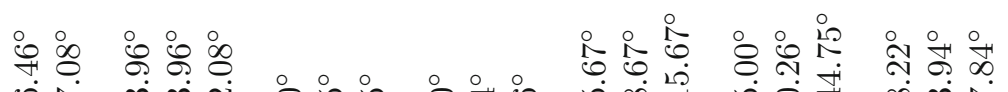

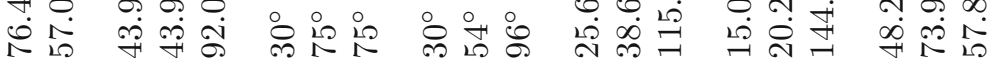

몽

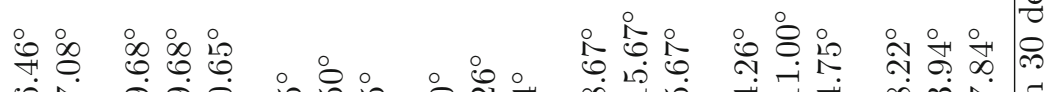
î

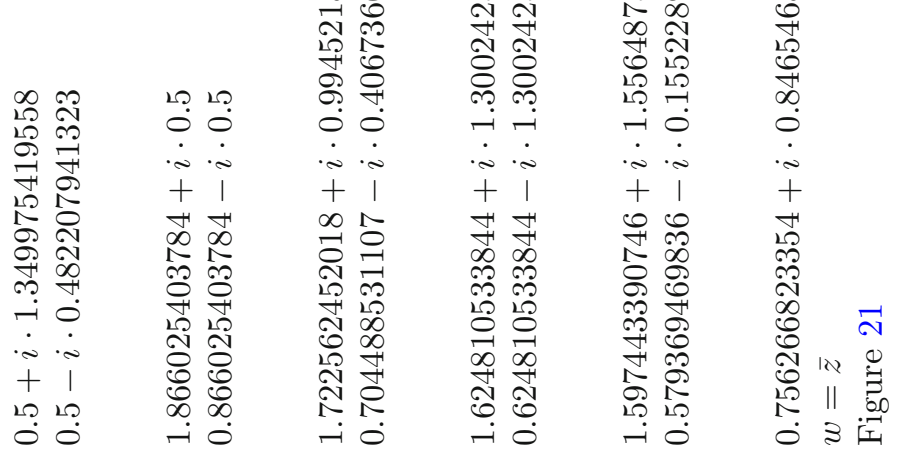

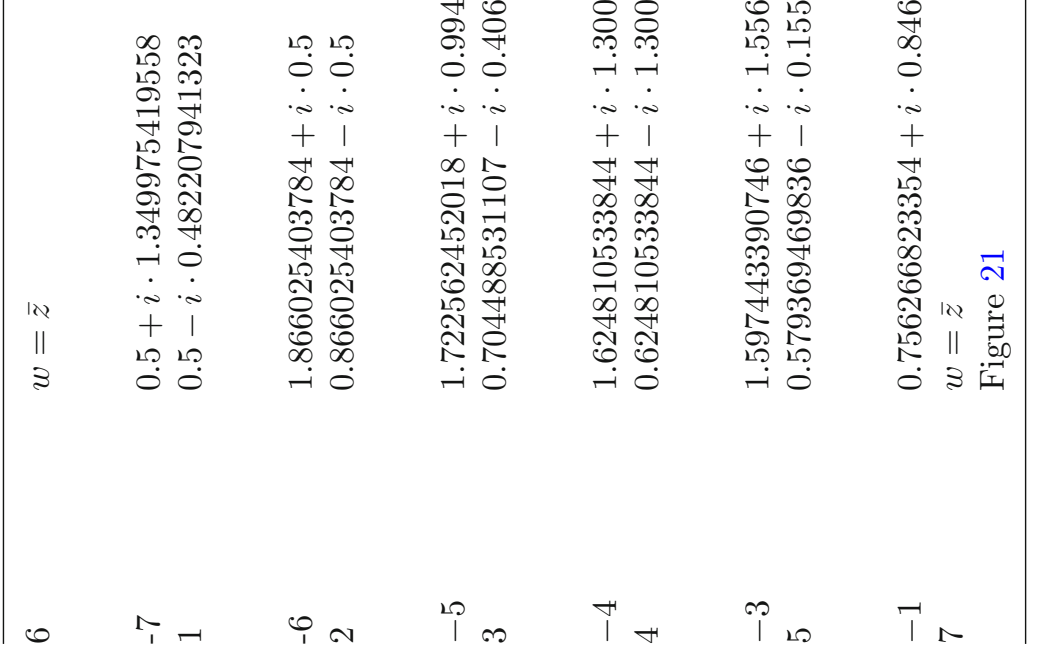


The face pairing generators will be

$$
\begin{array}{ll}
\mathbf{z}_{1}: \infty z 0 \rightarrow \infty 0 w & \Longrightarrow u \mapsto \frac{\bar{u} w-w \bar{z}}{-\bar{z}}, \\
\mathbf{z}_{1}:(u, 1) \mapsto \overline{(u, 1)}\left(\begin{array}{cc}
w & 0 \\
-w \bar{z}-\bar{z}
\end{array}\right), & \mathbf{z}_{1}^{-1}:(u, 1) \mapsto \overline{(u, 1)}\left(\begin{array}{cc}
-z & 0 \\
\bar{w} z & \bar{w}
\end{array}\right) ; \\
\mathbf{z}_{2}: \infty w 1 \rightarrow \infty 1 z & \Longrightarrow u \mapsto \frac{\bar{u}(z-1)+1-\bar{w} z}{1-\bar{w}}, \\
\mathbf{z}_{2}:(u, 1) \mapsto \overline{(u, 1)}\left(\begin{array}{ll}
z-1 & 0 \\
1-\bar{w} z & 1-\bar{w}
\end{array}\right), & \mathbf{z}_{2}^{-1}:(u, 1) \mapsto \overline{(u, 1)}\left(\begin{array}{cc}
1-w \bar{z}-1 & 0 \\
w-1
\end{array}\right) ; \\
\mathbf{s}: z 10 \rightarrow 10 w & \Longrightarrow u \mapsto \frac{u z w-z w}{u(w z-w+1)-z}, \\
\mathbf{s}:(u, 1) \mapsto(u, 1)\left(\begin{array}{ll}
z w & w z-w+1 \\
-z w-z & -z
\end{array}\right), & \mathbf{s}^{-1}:(u, 1) \mapsto(u, 1)\left(\begin{array}{ll}
-z-w z+w-1 \\
z w & z w
\end{array}\right) ; \\
\mathbf{s}^{2}:(u, 1) \mapsto(u, 1)\left(\begin{array}{cc}
w & w(z-1)+1 \\
-z w-w(z-1)-z
\end{array}\right) ; &
\end{array}
$$

as mappings of the complex projective line $\mathbb{C}_{\infty}, \mathbf{z}_{1}$ and $\mathbf{z}_{2}$ reverse the orientation, s preserves it. The cycle relations to the edge classes

$$
\longrightarrow: \mathbf{1}=\mathbf{z}_{1}^{2} \mathbf{z}_{2}^{2}, \quad \Longrightarrow: \mathbf{s}^{2} \mathbf{z}_{1}^{-1} \mathbf{s z}_{2}=\mathbf{1} \text { imply }|z||1-w|=|w||1-z|=1
$$

equivalently by (3.5) and straightforward computations, with care on matrix conjugation. We can treat the cusps, indicated by $\circ$ and $\bullet$, respectively, by gluing the vertex domains to a fundamental polygon on $\mathbb{C}_{\infty}$ for each cusp. Our gluing algorithm led to a fundamental domain of the Euclidean plane group 4.pg in each case. The two generating glide reflections can be read off the unglued paired sides.

For the cusp $\circ$ we have only one vertex domain of $A=\infty$, with the group pg, generated by $\mathbf{z}_{1}$ and $\mathbf{z}_{2}$ in (3.5). The surgery condition requires a finite order for the oriented "rotation" $\mathbf{z}_{1} \mathbf{z}_{2}$ about an axis $v \infty$ in the model:

$$
\begin{aligned}
& \mathbf{z}_{1} \mathbf{z}_{2}=\mathbf{z}_{1} \mathbf{s}^{-1} \mathbf{z}_{1} \mathbf{s}^{-2}:(u, 1) \mapsto(u, 1)\left(\begin{array}{cc}
\bar{w} & 0 \\
-\bar{w} z-z
\end{array}\right)\left(\begin{array}{ll}
z-1 & 0 \\
1-\bar{w} z & 1-\bar{w}
\end{array}\right) \\
& =(u, 1)\left(\begin{array}{l}
\bar{w}(z-1) 0 \\
(\bar{w}-1) z z(\bar{w}-1)
\end{array}\right) .
\end{aligned}
$$

Denote by $\epsilon=e^{ \pm i 2 \pi \bar{p} / p}$ the "signed rotational parameter" by (3.6), for the above situation, indicated also in upper right Fig. 13 (analogous to $\kappa=$ $e^{ \pm i 2 \pi \cdot l / k}$ in Sect. 2, Remark 2.1). Then $\left(\mathbf{z}_{1} \mathbf{z}_{2}\right)^{p}=\mathbf{1}$ and we get the first equation for $z, w$ :

$$
\frac{\bar{w}}{\bar{w}-1} \cdot \frac{z-1}{z}=\epsilon:=e^{ \pm i 2 \pi \bar{p} / p} \quad \text { for } \quad p=1,2,3, \ldots \text { and } \bar{p}=0, \ldots\lfloor p / 2\rfloor \text {. }
$$

More geometrically, $\arg (z-1) / z=\alpha_{2}=\arg \epsilon+\arg (\bar{w}-1) / \bar{w}$ (see also (2.11), Table 7 and Figs. 22, 23 and 24. 
We select the above $\epsilon$ and the next $\phi$ by the obvious freedom, first for simple tiling:

$$
\operatorname{Im} z>0, \quad \operatorname{Im} w<0 .
$$

For the cusp $\bullet$ we glue the 4 vertex domains to $B=0$. The images $E^{\star}:=E^{\mathbf{s}^{-1}}$, $C^{\star}:=C^{\mathbf{s}}$ and $D^{\star}=D^{\text {ss }}$ (Fig. 13) show us the generating glide reflections: $\mathbf{g}_{1}$ and $\mathbf{g}_{2}$ :

$$
\begin{array}{ll}
\mathbf{g}_{1}:\left[z_{1}^{-1}\right] \mapsto\left[z_{1}\right]^{\mathbf{s}^{-1}} \Rightarrow & \mathbf{g}_{1}=\mathbf{z}_{1} \mathbf{s}^{-1}, \\
\text { or }\left[z_{2}^{-1}\right]^{\mathbf{s}^{-1}} \mapsto\left[z_{2}^{\mathbf{s}} \Rightarrow \mathbf{g}_{1}=\mathbf{s z}_{2} \mathbf{s},\right. & \\
\mathbf{g}_{2}:\left[z_{2}\right]^{\mathbf{s s}} \mapsto\left[z_{2}^{-1}\right]^{\mathbf{s}} \Rightarrow & \\
\text { or }\left[z_{1}\right] \mapsto\left[z_{1}^{-1}\right]^{\mathbf{s s}} \Rightarrow \mathbf{g}_{2}=\mathbf{z}_{1}^{-1} \mathbf{s}^{2} . &
\end{array}
$$

We need also a "signed rotation parameter" $\phi=e^{ \pm i 2 \pi \bar{q} / q}$ for $\mathbf{g}_{1} \mathbf{g}_{2}^{-1}$ $=\mathbf{z}_{1} \mathbf{s}^{-2} \mathbf{z}_{2} \mathbf{s}^{2}=\mathbf{z}_{1} \mathbf{s}^{-3} \mathbf{z}_{1}$ and by (3.5) we get:

$$
\mathbf{g}_{1} \mathbf{g}_{2}^{-1}:(u, 1) \mapsto(u, 1)\left(\begin{array}{ll}
w(1-\bar{z}) & (\bar{w}-\bar{z})[1-w(1-z)] \\
0 & z(1-\bar{w})
\end{array}\right) .
$$

Hence $\left(\mathbf{g}_{1} \mathbf{g}_{2}^{-1}\right)^{q}=\mathbf{1}$ yields the second equation for $z, w$ :

$$
\begin{gathered}
\frac{w}{1-\bar{w}} \cdot \frac{1-\bar{z}}{z}=\phi:=e^{ \pm i 2 \pi \bar{q} / q} \quad \text { for } q=1,2,3,4, \ldots, \\
\text { and } \bar{q}=0, \ldots,\lfloor q / 2\rfloor
\end{gathered}
$$

since $|z(1-\bar{w})|=1=|w(1-\bar{z})|$ holds by (3.6). That means, we do not get further manifold surgery here. We can express, say $w$ from (3.8) and carefully reduce the (implicite, left to computer) equations for $w$ and $z$ :

$$
w=\frac{\bar{\epsilon} \bar{z}}{(\bar{\epsilon}-1) \bar{z}+1}, \quad \frac{z(1-z)}{(\epsilon-1) z+1}= \pm \sqrt{\bar{\epsilon} \bar{\phi}}, \quad \operatorname{Im} z>0>\operatorname{Im} w, \operatorname{first}(3
$$

If $\epsilon=1, p=1$ in (3.8), i.e. $w=\bar{z}$, the transform $\mathbf{z}_{1} \mathbf{z}_{2}$ will be a translation $u \rightarrow u+1$ by (3.7) with the only fixed point $\infty$ of $\mathbb{C}_{\infty}$. Then the cusp $\circ$ remains for the double-simplex.

Else we get the rotation axis, connecting

$$
v:=\frac{1}{1-\epsilon}=\frac{z(\bar{w}-1)}{\bar{w}-z}, \quad \infty,
$$

that has to be out of the vertex domain of $A(\infty)$ in upper right Fig. 13

$$
A(\infty)=[B(0), E(w), C(1), D(z)] .
$$

For an axis point $\lambda z$ at $z_{1}$ we get the equation $[\lambda z-v] /[(1-\lambda) w-v]=|z| /|w|$, or for translation $\mathbf{z}_{1} \mathbf{z}_{2}$ we get now $\lambda=1 / 2$ and parallel axes to the translation. Similarly, $\phi=1, \quad q=1$ leads to a parabolic transform $\mathbf{g}_{1} \mathbf{g}_{2}^{-1}$ in (3.10) with only fixed point 0 yielding a cusp in it. 
The other cases of $\phi$ involve corresponding rotation axes connecting the ends $0, y \in \mathbb{C}_{\infty}$ in the half-space model where

$$
y:=\frac{w(1-\bar{z})-z(1-\bar{w})}{(\bar{w}-\bar{z})[1-w(1-z)]}
$$

is the fixed point, by (3.10-3.11) depending on $\epsilon$ and $\phi$, that has to be out of the vertex domain of $B(0)$, as follows

$$
\begin{aligned}
B(0) & =\left[E(w), A(\infty), D(z), A^{\mathbf{s}^{-1}}\left(\frac{z}{w z-w+1}\right), C(1), A^{\mathbf{s}}\left(\frac{z w}{w z-w+1}\right),\right. \\
E^{\mathbf{s}} & \left.=B^{\mathbf{s s}}\left(\frac{w z}{w z-w+1}\right), A^{\mathbf{s s}}\left(\frac{w}{w z-w+1}\right)\right] .
\end{aligned}
$$

A reciprocity transform $u \mapsto \frac{1}{u}, 0 \mapsto \infty$ will help us in deciding this criterion in lower right Fig. 13. Indeed we have convenient $y \rightarrow 1 / y$ for $\epsilon$ and $\phi$, furthermore for $z$ and $w$ and analogous transforms $\overline{\mathbf{g}}_{1}$ and $\overline{\mathbf{g}}_{2}$.

The axis point for $\overline{\mathbf{g}}_{1}$ on $A D A^{\mathbf{s}^{-1}}$ and for $\overline{\mathbf{g}}_{2}$ on $A E A^{\mathbf{s s}}$, respectively, can be obtained analogously as before (easy by computer, formulas are not easy).

Now, the logical symmetries $D \leftrightarrow E$ and (independently) $B \leftrightarrow C$ of the double-simplex $\widetilde{\mathcal{D}}_{1}$ in Fig. 13 allow us to consider only some essentially different cases, say, with simplified conventions, now for $\bar{p}=1$ and $\bar{q}=1$ to the possible orbifold cases and simple tilings, first:

$$
\epsilon=e^{i 2 \pi / p}, \quad 0<-p \in \mathbf{N}, \quad-p \neq 2 ; \quad \phi=e^{i 2 \pi / q}, \quad 0<q \in \mathbf{N} .
$$

Formulas (3.8) and (3.12-3.13) yield second degree equation for $z$, then we get unique $w$ of $z$. The discussion is easier with computer, indeed.

Some results are collected in our Table 7 in the above sense. See also our Figs. 19, 20, 21, 22, 23 and 24, the last one for multiple tilings, i.e. cone manifolds in Table 8 as well.

Remark 3.1. As at the Gieseking manifold in the previous section, we can glue the 2 cusps of $M_{c^{2}}$ with 2 solid Klein-bottles. Then we obtain a (nongeometrizable!) compact nonorientable manifold $\mathcal{M}$ whose fundamental group $\mathcal{G}_{\mathcal{M}}$ has a short presentation by two generators $\mathbf{z}_{1}=: \mathbf{z}$ and $\mathbf{s}$

$$
\mathcal{G}_{\mathcal{M}}=\left(\mathbf{z}, \mathbf{s}-\mathbf{1}=\mathbf{z}^{2} \mathbf{s}^{-1} \mathbf{z s}^{-3} \mathbf{z s}^{-2}\right) \text {. }
$$

This can be obtained if we take the two relations from (3.6), and eliminate $\mathbf{z}_{2}$. Geometrically see Fig. 18a.

The surgery conditions of the two cusps $\circ$ and $\bullet$ yield formally, by (3.8) and (3.12)

$$
\left(\mathbf{z s}^{-1} \mathbf{z s}^{-2}\right)_{\bar{p}}^{p}=\mathbf{1}=\left(\mathbf{z s}^{-3} \mathbf{z}\right)_{\bar{q}}^{q},
$$

respectively.

Remark 3.2. For fixed $(p, \bar{p})$, if $q \rightarrow \infty$, our double simplex and its tilings tend to cases with $q= \pm 1$. For fixed $(q, \bar{q})$, if $p \rightarrow-\infty$, our limit cases will be with 

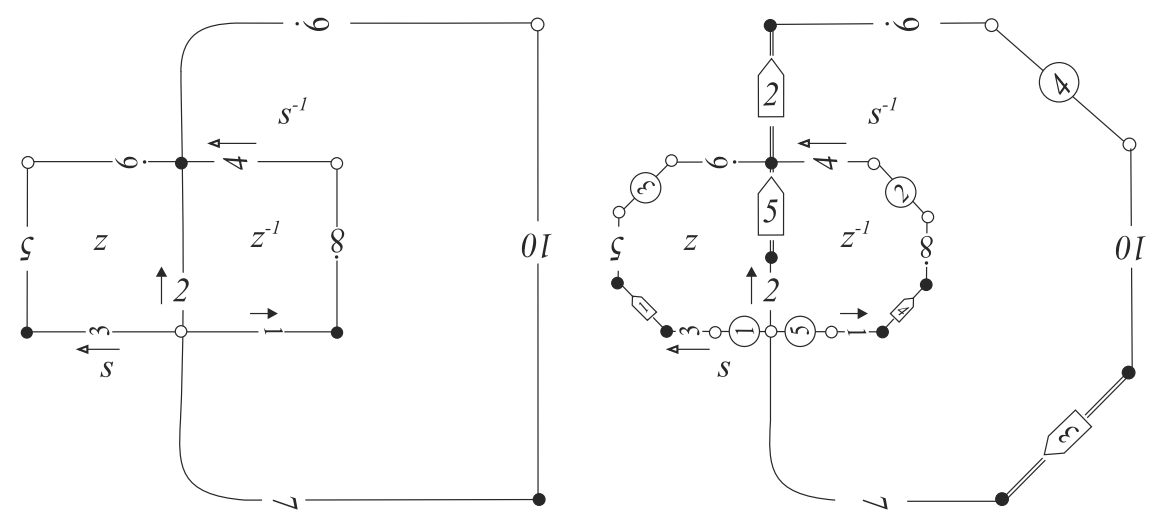

Figure 18 A combinatorial ( $\mathrm{PL}=$ piecewise linear) fundamental domain by Schlegel diagram a. for manifold $\widetilde{\mathcal{D}}_{1}$ b. and that for its specific orbifolds and cone manifolds

$p= \pm 1$. Both statements follow from (3.8), (3.12) and (3.13). Consequently, $p \rightarrow-\infty, q \rightarrow \infty$ leads to the regular case $( \pm 1, \pm 1)$.

Again, we summarize our results in

Theorem 3.3. The surgery of our hyperbolic nonorientable double simplex manifold $\widetilde{\mathcal{D}}_{1}$ with 2 cusps by two solid Klein-bottles leads to a nongeometrizable compact manifold $\mathcal{M}$ described in Fig. 18a with fundamental group by (3.19). We do not get any compact hyperbolic manifold.

With special surgery parameters $z(p, q), w(p, q)$ in Table 7 and formula (3.18), we get compact orbifolds with hyperbolic crystallographic group

$$
\mathcal{G}_{1}(p, q)=\left(\mathbf{z}_{1}, \mathbf{z}_{2}, \mathbf{s}-\mathbf{1}=\mathbf{z}_{1}^{2} \mathbf{z}_{2}^{2}=\mathbf{s}^{2} \mathbf{z}_{1}^{-1} \mathbf{s} \mathbf{z}_{2}=\left(\mathbf{z}_{1} \mathbf{z}_{2}\right)^{p}=\left(\mathbf{z}_{1} \mathbf{s}^{-2} \mathbf{z}_{2} \mathbf{s}^{2}\right)^{q}\right) .
$$

This $\widetilde{\mathcal{D}}_{1}(p, q)$ will have 2 closed geodesic lines with singularity points of order $p, q$, respectively (see Fig. 18b).

A lot of parameter pairs $(p, \bar{p}),(q, \bar{q})$ by (3.8), (3.12) and computer solution of (3.13) for $z$ and $w$ provide us with cone manifolds and multiple tilings with formal minimal group presentation by (3.19-3.20), Fig. 18b:

$$
\mathcal{G}_{1}[(p, \bar{p}),(q, \bar{q})]=\left(\mathbf{z}, \mathbf{s}-\mathbf{1}=\mathbf{z}^{2} \mathbf{s}^{-1} \mathbf{z s}^{-3} \mathbf{z s}^{-2}=\left(\mathbf{z s}^{-1} \mathbf{z s}^{-2}\right)_{\bar{p}}^{p}=\left(\mathbf{z s}^{-3} \mathbf{z}\right)_{\bar{q}}^{q}\right) .
$$

The cases $p= \pm 1$ or $q= \pm 1$ above, lead to one remaining cusp.

For this combinatorial (PL) graphic presentation see also $[12,17,20]$. We did not obtain zero limit volumes at surgeries of $\widetilde{\mathcal{D}}_{1}$. For its orbifolds ("crystallographic groups" or "lattices") we get the minimal volume $V=0.7177 \ldots$ at 

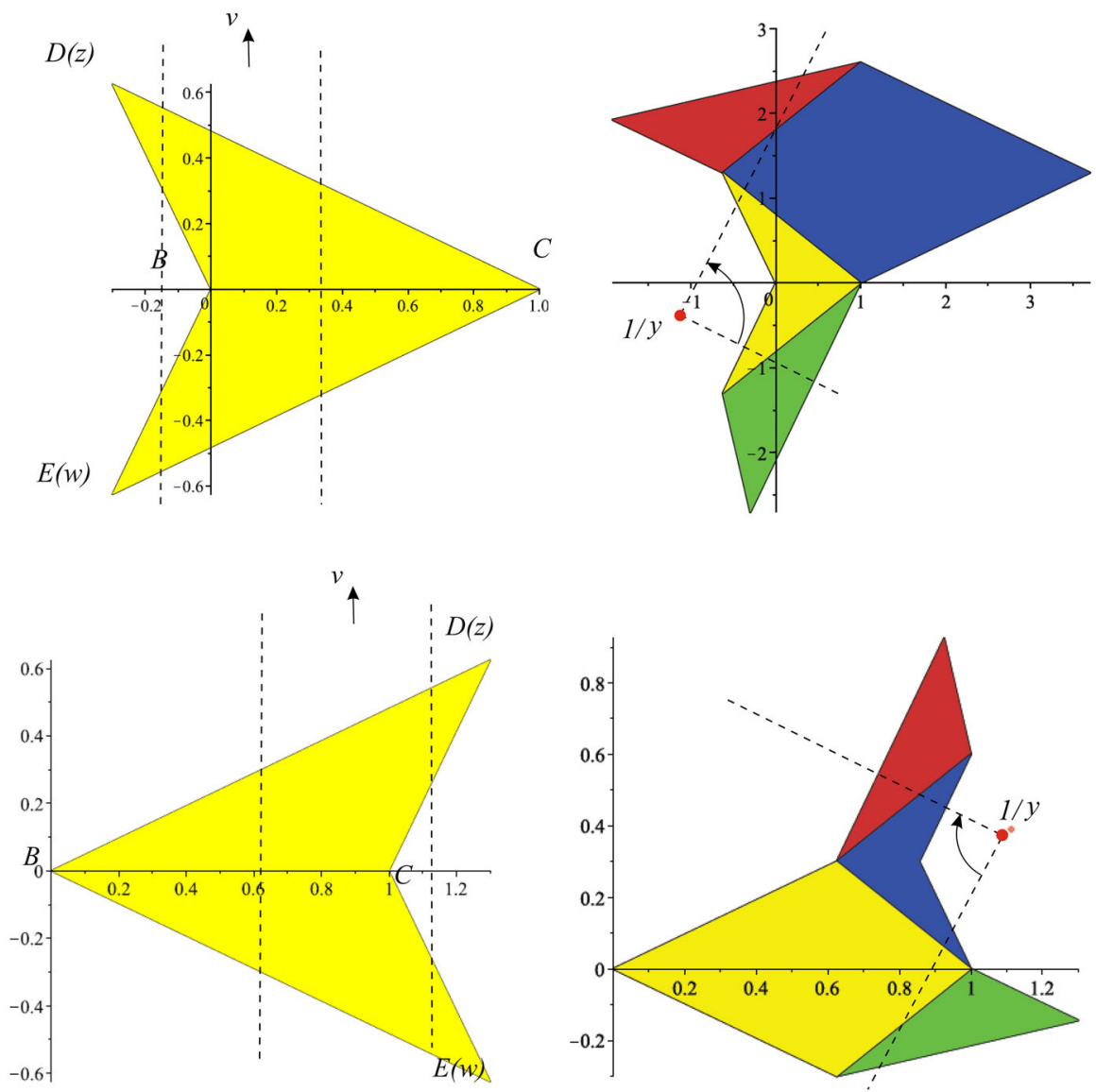

Figure $19(p, q)=(-1,-2)$ and $(p, q)=(-1,2)$ are equivalent (congruent)

$(p, q)=( \pm 3, \pm 2)$ (Table 7 , Fig. 22$)$, that we can prove by careful computer analysis. Multiple tilings, i.e. cone manifolds produce smaller volume, e.g. at $(-7,3),(3,1)$ (Fig. 24 in Table 8). 

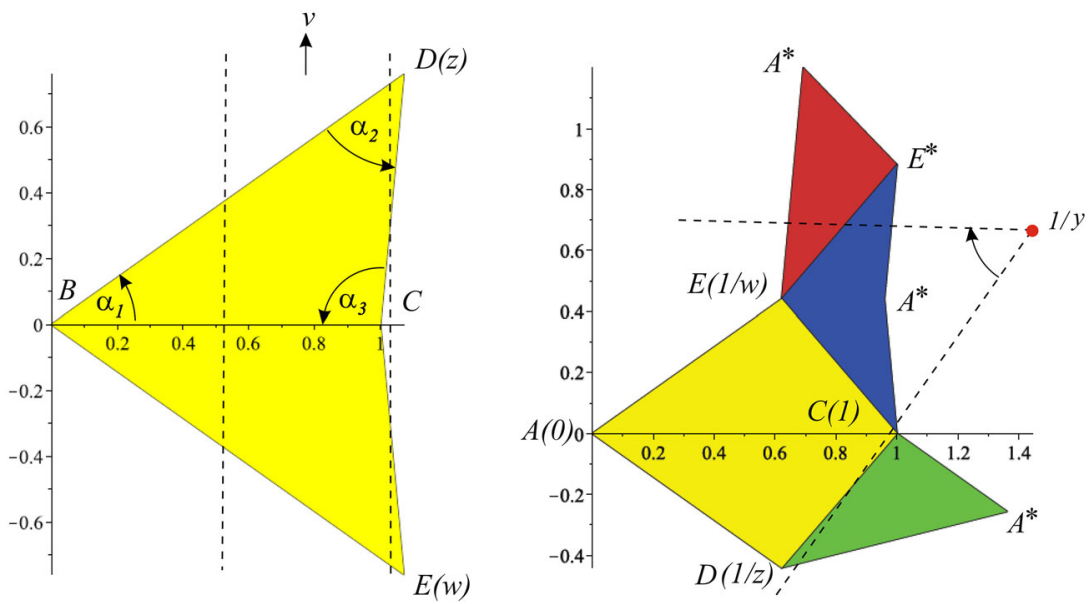

Figure $20(p, q)=(-1,3), \alpha_{1}=\arg z, \alpha_{2}=\arg (z-1) / z$, $\alpha_{3}=\arg 1 /(1-z)$ (see Table 7$)$
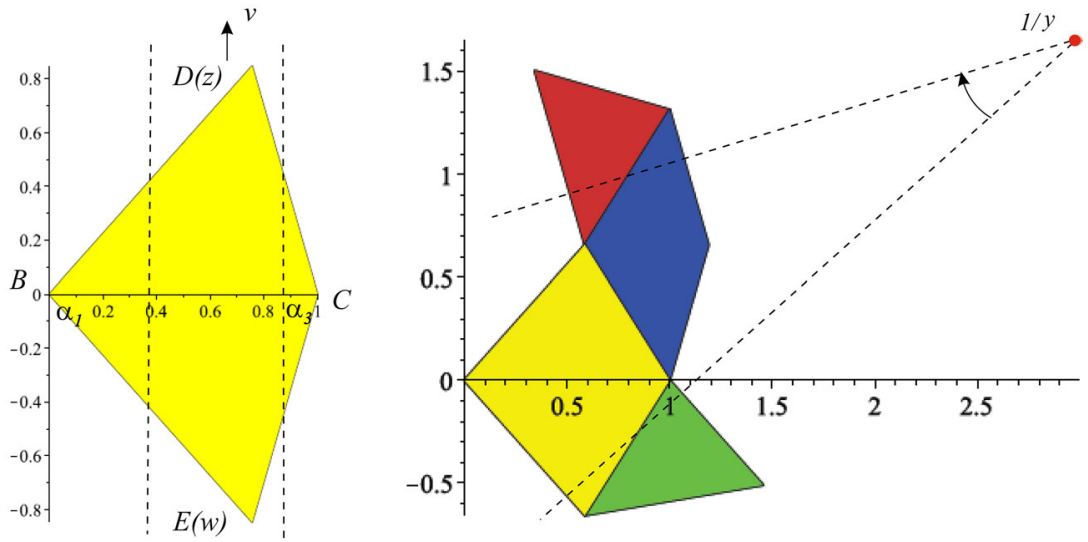

Figure $21(p, q)=(-1,7)$ 

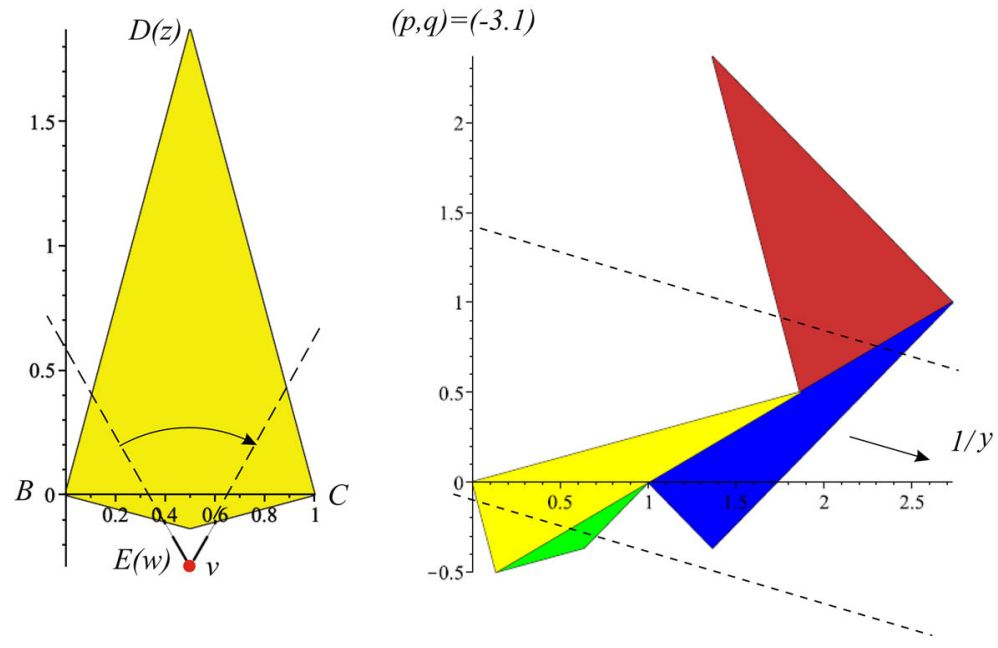

$(p, q)=(-3.2)$
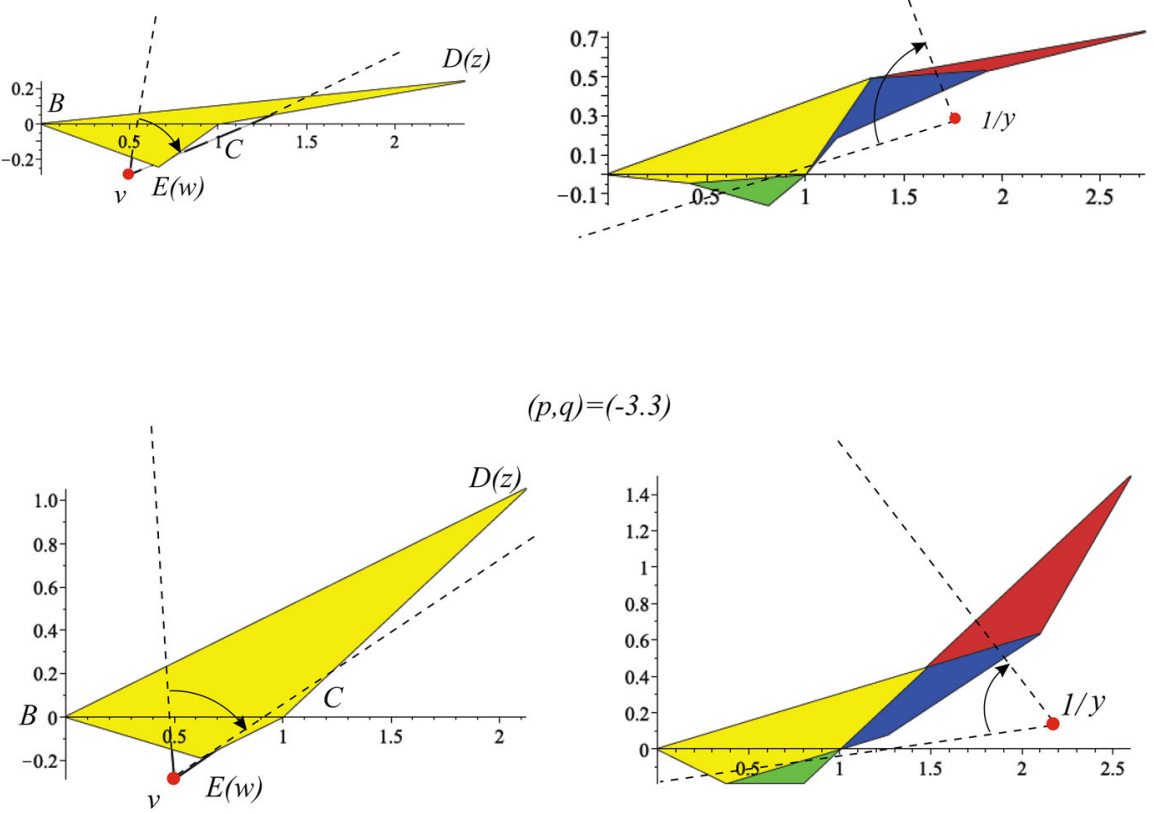

$(p, q)=(-3.3)$

FiguRE 22 Nice orbifolds 
$(p, q)=(-4,1)$
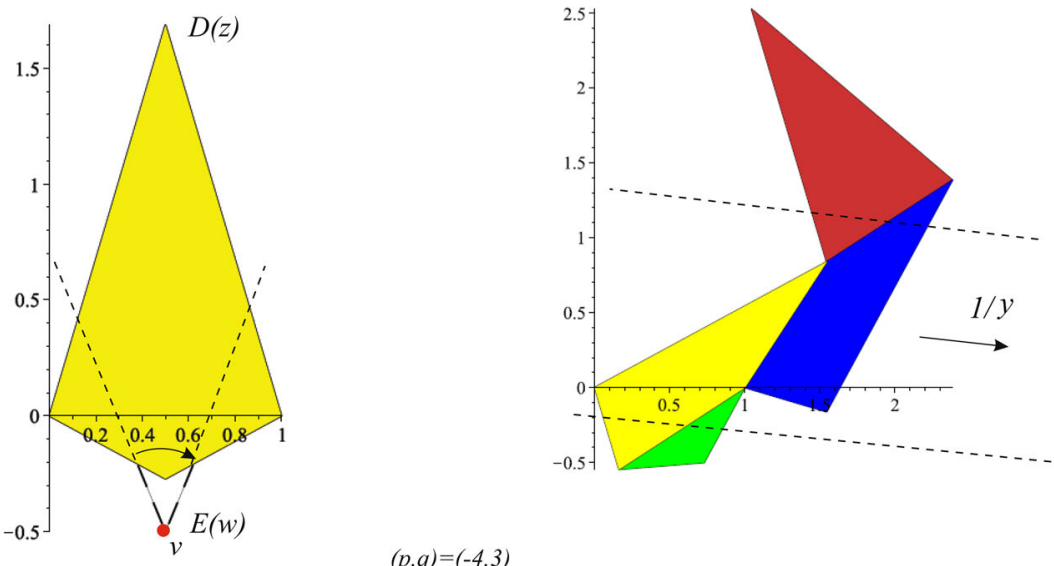

$(p, q)=(-4,3)$
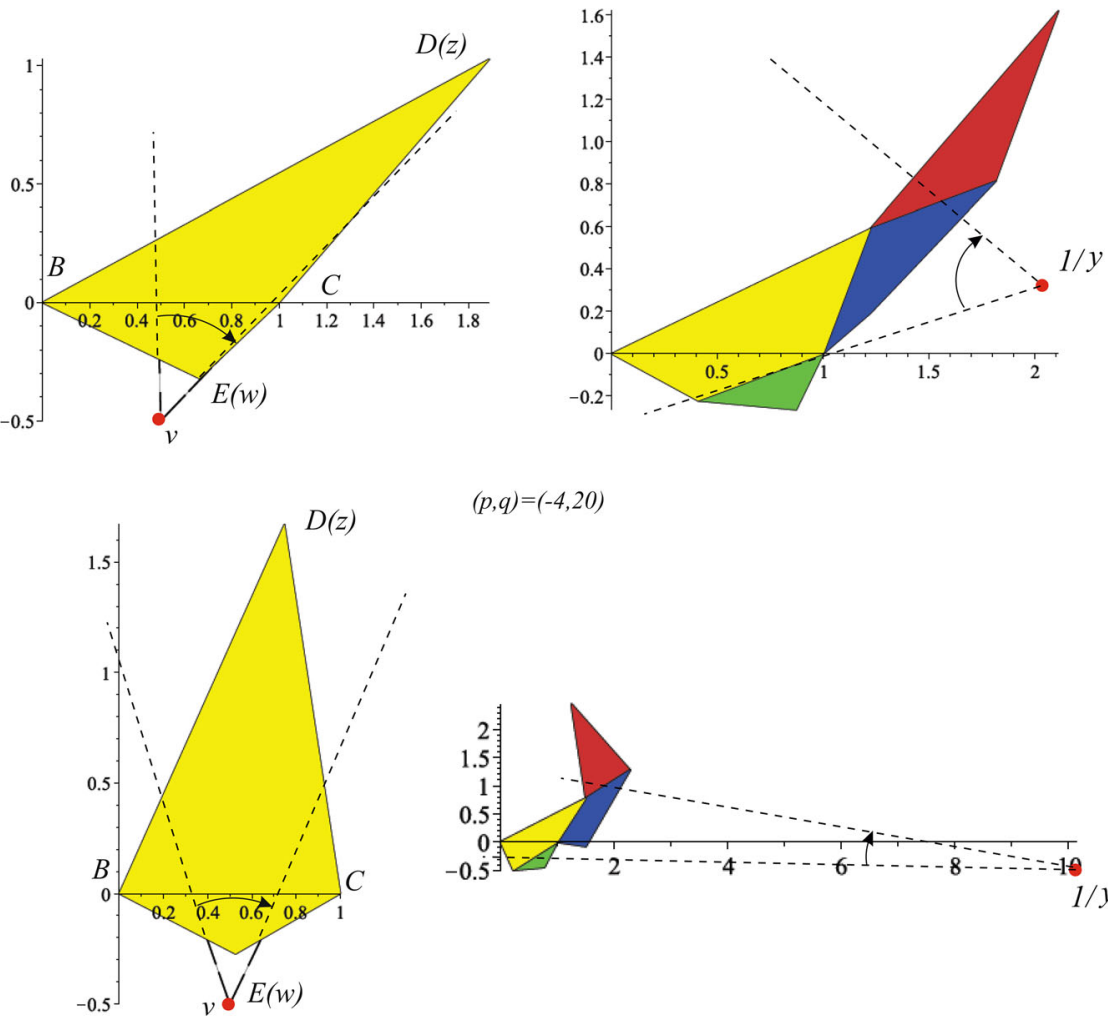

$(p, q)=(-4,20)$

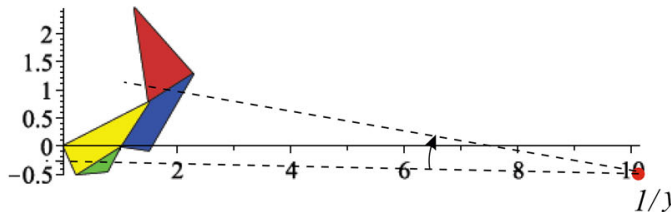

Figure 23 Orbifolds $(p, q)=(-4,20) \rightarrow(p, q)=(-4,1)$ indicates the limit case $q \rightarrow \infty$ 
$(p, \bar{p})=(-3,1),(q, \bar{q})=(5,2)$
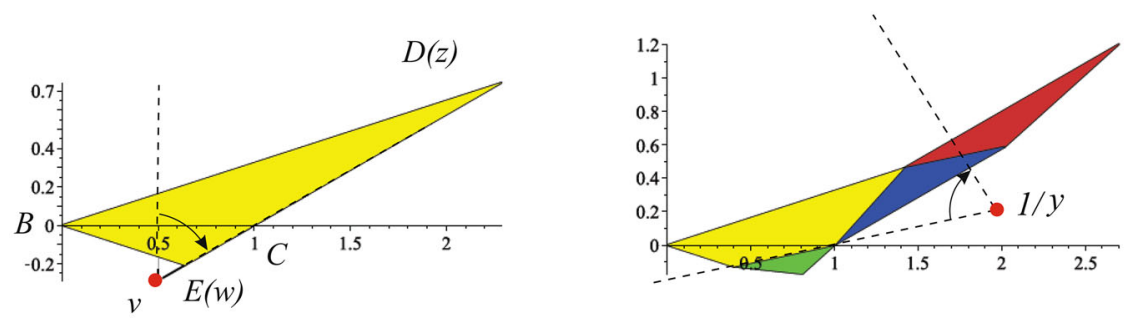

$(p, \bar{p})=(-7,3),(q, \bar{q})=(3,1)$
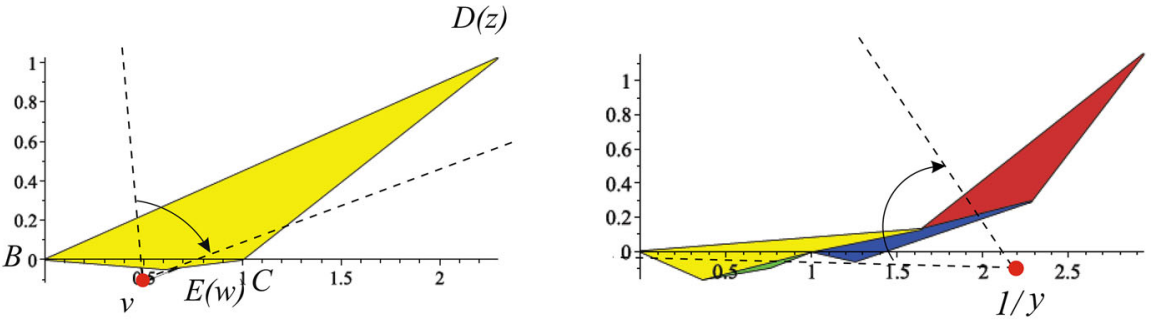

$(p, \bar{p})=(-5,1),(q, \bar{q})=(7,2)$
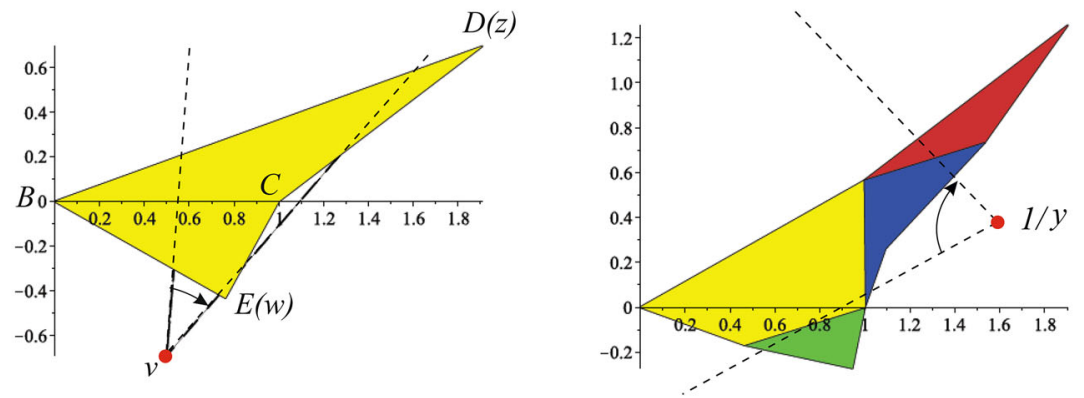

FIGURE 24 Multiple tilings, i.e. cone manifolds 


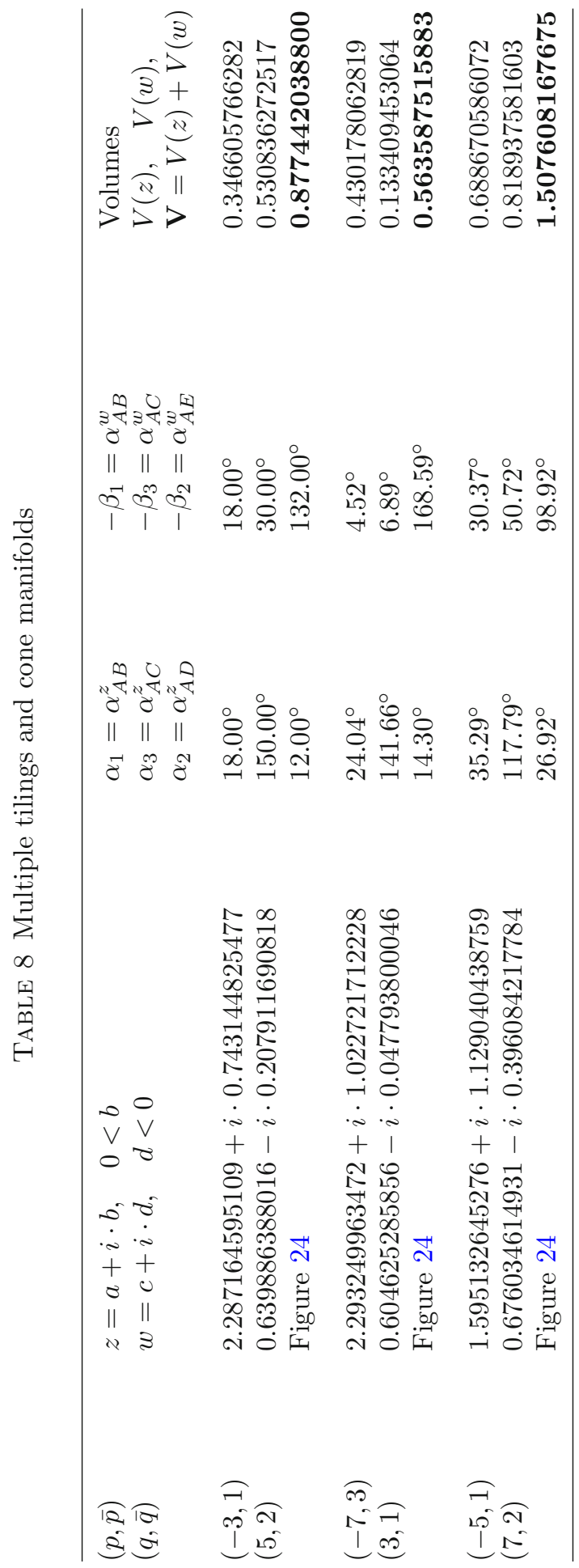


$(p, q)=(4,-3)$
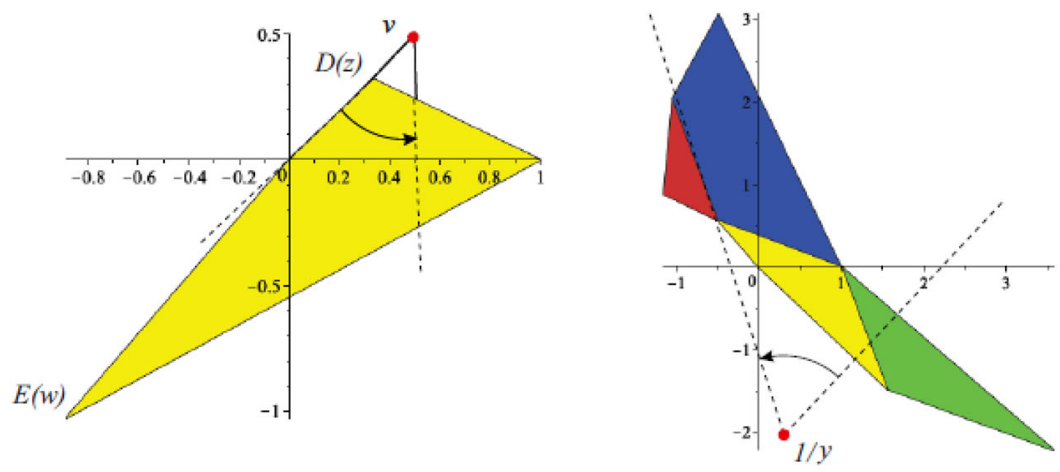

$(p, q)=(4,3)$-non-realizable

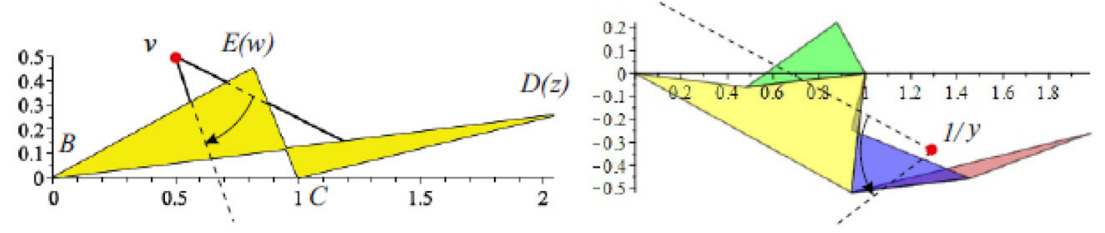

$(p, \bar{p})=(-3,2)(q, \bar{q})=(5,2)$-non-realizable

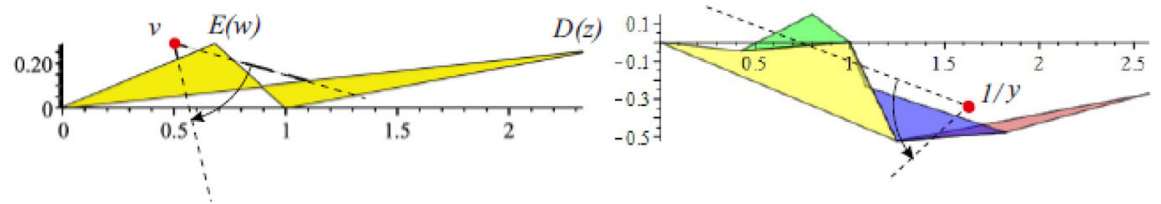

FiguRE 25 Extra cases 
Funding Open Access funding provided by Budapest University of Technology and Economics

Open Access. This article is licensed under a Creative Commons Attribution 4.0 International License, which permits use, sharing, adaptation, distribution and reproduction in any medium or format, as long as you give appropriate credit to the original author(s) and the source, provide a link to the Creative Commons licence, and indicate if changes were made. The images or other third party material in this article are included in the article's Creative Commons licence, unless indicated otherwise in a credit line to the material. If material is not included in the article's Creative Commons licence and your intended use is not permitted by statutory regulation or exceeds the permitted use, you will need to obtain permission directly from the copyright holder. To view a copy of this licence, visit http://creativecommons. org/licenses/by/4.0/.

Publisher's Note Springer Nature remains neutral with regard to jurisdictional claims in published maps and institutional affiliations.

\section{References}

[1] Adams, C.: Volumes of n-cusped hyperbolic 3-manifolds. J. Lond. Math. Soc. (2) 38, 555-565 (1988)

[2] Adams, C., Sherman, W.: Minimum ideal triangulations of hyperbolic 3manifolds. Discret. Comput. Geom. 6, 135-153 (1991)

[3] Cavicchioli, A., Molnár, E., Telloni, A.I.: Some hyperbolic space forms with few generated fundamental groups. J. Korean Math. Soc. 50(2), 425-444 (2013)

[4] Cavicchioli, A., Spaggiari, F.: Tetrahedron manifold series of Heegaard genus two with knot presentation and Dehn surgery. Acta Math. Hung. 131(4), 307322 (2011)

[5] Fomenko, A.T., Matveev, S.V.: Isoenergetic surfaces of Hamiltonian systems, account of three-dimensional manifolds in order of their complexity and computation of volumes of closed hyperbolic manifolds. Uspehi Mat. Nauk 43, 5-22 (1988). (Russian)

[6] Fomenko, A.T., Matveev, S.V.: Algorithmic and Computer Methods for ThreeManifolds (Mathematics and Its Applications), vol. 425. Kluwer Academic Publishers, Dordrecht (1997)

[7] Francis, J.: Three-manifolds obtainable from two and three tetrahedra. Master Thesis, William College (1987)

[8] Kazhdan, D.A., Margulis, G.A.: A proof of Selberg's hypothesis. Mat. Sb. (N.S.) 75/117, 163-168 (1968)

[9] Marshall, T.H., Martin, G.J.: Minimal co-volume hyperbolic lattices, II: simple torsion in a Kleinian group. Ann. Math. 176, 261-301 (2012) 
[10] Mednykh, A.D.: Volumes of two-bridge cone manifolds in spaces of constant curvature. Transform. Groups (2020). https://doi.org/10.1007/s00031-020-09632-x. (to appear)

[11] Molnár, E., On isometries of space forms. Colloquia Math. Soc. János Bolyai 56 (1989). Differential Geometry and Its Applications, Eger (Hungary), pp. 509534. North-Holland Co., Amsterdam (1992)

[12] Molnár, E.: Polyhedron complexes with simply transitive group actions and their realizations. Acta Math. Hung. 59(1-2), 175-216 (1992)

[13] Molnár, E.: Discontinuous groups in homogeneous Riemannian spaces by classification of D-symbols. Publ. Math. Debr. 49(3-4), 265-294 (1996)

[14] Molnár, E.: The projective interpretation of the eight 3-dimensional homogeneous geometries. Beiträge Algebra Geom. 38/2, 261-288 (1997)

[15] Molnár, E., Prok, I., Szirmai, J.: The Gieseking manifold and its surgery orbifolds. Novi Sad J. Math. 29(3), 187-197 (1999)

[16] Molnár, E., Prok, I., Szirmai, J.: Classification of hyperbolic manifolds and related orbifolds with charts up to two ideal simplices. In: Karáné G., Sachs H., Schipp F. (eds.) Proceedings of "Internationale Tagung über Geometrie, Algebra und Analysis", Strommer Gyula Nemzeti Emlékkonferencia, Balatonfüred Budapest, Hungary, pp. 293-315 (1999)

[17] Molnár, E., Prok, I., Szirmai, J.: Classification of tile-transitive 3-simplex tilings and their realizations in homogeneous spaces. In: Prékopa, A., Molnár, E. (eds.) Non-Euclidean Geometries, János Bolyai Memorial Volume Mathematics and Its Applications, vol. 581, pp. 321-363. Springer (2006)

[18] Molnár, E., Prok, I., Szirmai, J.: Surgeries of the Gieseking hyperbolic ideal simplex manifold. Publ. Math. Debr. (2020) (Accepted)

[19] Molnár, E., Szirmai, J., Week, J.: 3-Simplex tilings, splitting orbifolds and manifolds. Symmetry Cult. Sci. 22(3-4), 435-459 (2011)

[20] Prok, I.: Data structures and procedures for a polyhedron algorithm. Period. Polytech. Ser. Mech. Eng. 36(3-4), 299-316 (1992)

[21] Prok, I.: On maximal homogeneous 3-geometries: a polyhedron algorithm for space tilings. Universe MDPI 4(49), 4030049 (2018). https://doi.org/10.3390/ universe

[22] Ranicki, A.A., Wall, C.T.C. (eds.): Surgery on Compact Manifolds, 2nd edn. American Mathematical Society, Providence (1998)

[23] Thurston, W.: The Geometry and Topology of 3-Manifolds (Lecture Notes). Princeton University, Princeton (1978)

[24] Vinberg, E.B. (ed.): Geometry II: Spaces of Constant Curvature. Spriger, Berlin (1993)

[25] Weeks, J.R.: Hyperbolic structures on three-manifolds. PhD dissertation, Princeton (1985)

[26] Wolf, J.A.: Spaces of Constant Curvature. University of California, Berkeley (1972) 
E. Molnár, I. Prok and J. Szirmai Department of Geometry

BME MI

P.O. Box 91

Budapest 1521

Hungary

e-mail: szirmai@math.bme.hu

E. Molnár

e-mail: emolnar@math.bme.hu

I. Prok

e-mail: prok@math.bme.hu

Received: October 5, 2020.

Accepted: December 9, 2020. 